PHYSICAL REVIEW D 72, 084009 (2005)

\title{
Semirelativistic approximation to gravitational radiation from encounters with nonspinning black holes
}

\author{
Jonathan R. Gair* \\ Theoretical Astrophysics, California Institute of Technology, Pasadena, California 91125, USA \\ Daniel J. Kennefick ${ }^{\dagger}$ \\ Theoretical Astrophysics, California Institute of Technology, Pasadena, California 91125, USA, \\ and Department of Physics, University of Arkansas, Fayetteville, Arkansas 72701, USA
}

Shane L. Larson

Center for Gravitational Wave Physics, Pennsylvania State University, University Park, Pennsylvania 16802, USA

(Received 11 August 2005; published 13 October 2005)

\begin{abstract}
The capture of compact bodies by black holes in galactic nuclei is an important prospective source for low frequency gravitational wave detectors, such as the planned Laser Interferometer Space Antenna. This paper calculates, using a semirelativistic approximation, the total energy and angular momentum lost to gravitational radiation by compact bodies on very high eccentricity orbits passing close to a supermassive, nonspinning black hole; these quantities determine the characteristics of the orbital evolution necessary to estimate the capture rate. The semirelativistic approximation improves upon treatments which use orbits at Newtonian order and quadrupolar radiation emission, and matches well onto accurate Teukolsky simulations for low eccentricity orbits. Formulas are presented for the semirelativistic energy and angular momentum fluxes as a function of general orbital parameters.
\end{abstract}

DOI: 10.1103/PhysRevD.72.084009

PACS numbers: 04.30.Db

\section{INTRODUCTION}

Proposed space-based gravitational wave interferometers such as the Laser Interferometer Space Antenna (LISA) will have good sensitivities in the low frequency gravitational wave band, from about $10^{-4} \mathrm{~Hz}$ up to about $1 \mathrm{~Hz}$. A promising source of gravitational waves in this band is the extreme-mass-ratio inspiral (EMRI) of compact objects (stellar mass black holes, neutron stars, white dwarfs and even main sequence stars) into massive black holes. Current estimates suggest we might detect as many as a thousand EMRI events over the course of the LISA mission [1]. Gravitational waves from extreme-mass-ratio captures will serve as a direct probe of the innermost population of compact objects around galactic central black holes, and also provide information on the growth history of such black holes out to a significant redshift $(z \sim 1)$. In addition to probing the stellar population of galactic nuclei, gravitational waves from EMRIs provide a map which encodes the geometry and structure of the black hole spacetime [2], allowing a direct comparison of the astrophysical black hole to the black hole solutions of general relativity. This technique has been called "holiodesy," in analogy with satellite geodesy, which observes the

\footnotetext{
*Present address: Institute of Astronomy, Madingley Road, Cambridge, CB3 0HA, United Kingdom.

Electronic address: jgair@ast.cam.ac.uk

Electronic address: danielk@uark.edu

\#Electronic address: shane@gravity.psu.edu
}

motion of small satellites around the Earth to map out the structure of the planet's gravitational field.

The computation of gravitational radiation from stellar orbits has a long history. The classic treatment was that of Peters and Mathews [3,4], who computed the gravitational wave emission from stars on purely Keplerian orbits in flat space. The problem of generation and propagation of gravitational waves in a Kerr background was addressed by the work of Teukolsky and Press in the early 1970s [5-7], who developed a perturbation formalism in the Kerr background. Subsequent work using this formalism has progressed to the point where the emission from a particle on any orbit in the Schwarzschild spacetime [8] or on a circular inclined [9] or eccentric equatorial [10] orbit in the Kerr background can be treated. Computing the inspiral of stars on eccentric nonequatorial orbits in Kerr required overcoming some technical obstacles [9], but first results are now available [11,12].

In this paper we compute a star's orbital trajectory by solving the geodesic equations of motion around the black hole, rather than using Keplerian orbits. Exact geodesics of the Schwarzschild spacetime are considered, particularly orbits with high eccentricity, including marginally bound (parabolic) and unbound (hyperbolic) orbits. Here "parabolic" is a statement about the orbital energy $E$ which labels the geodesic trajectory, rather than a statement about the geometric shape of the orbit in Euclidean geometry. As will be seen in later sections, the orbital trajectories around black holes can exhibit "zoom-whirl" behavior, looping around the black hole more than once 
(i.e., the change in azimuthal angle $\Delta \phi>2 \pi$ ) on any given orbital pass. Even in these situations one can still speak of quantities, such as the eccentricity of the orbit, which correspond in some formal sense to their Keplerian counterparts. Taking these geodesic trajectories as the orbits of the source, we approximate the gravitational radiation using the classic quadrupole formula at Newtonian order.

This method of obliging the orbiting body to follow a geodesic of the spacetime, while using the quadrupole approximation to calculate the gravitational wave emission, has been termed the "semirelativistic approximation" by its originators, Ruffini and Sasaki [13]. Experience with more accurate, although computationally intensive, perturbative calculations has shown that when particles make close approaches to the central black hole, in particular, when they are relatively close to the unstable circular orbit (UCO) of the potential (see Sec. II B for a definition), the properties of the relativistic gravitational potential are of critical importance in determining the gravitational wave flux. As will be seen, employing a more exact description of the particle trajectory (spacetime geodesics) together with an approximation of the wave flux is much more accurate in these cases than using a consistent Newtonian-order approximation.

The semirelativistic approach complements the more complex Teukolsky-based computations in several ways. First, technical difficulties and the demands of computing power have made Teukolsky calculations computationally difficult for orbits with eccentricity near unity, a regime where the work presented here is designed to work well. Second, because the computationally intensive Teukolsky approach is not practical for use in conjunction with typical simulations of the clusters of stars in galactic centers and their capture rates by the central black hole, it is useful to look for more convenient approximate methods, which are sufficiently accurate for reliable results.

An extension of the semirelativistic approach is currently finding use in the computation of approximate EMRI waveforms for use in scoping out LISA data analysis [14-16]. A particle inspiral trajectory is computed by integrating post-Newtonian expressions for the energy and angular momentum fluxes. Integration of the Kerr geodesic equations along this trajectory yields the particle position as a function of time, from which a waveform is computed from an approximate quadrupole moment tensor generated as in the semirelativistic approach. These "numerical kludge" waveforms are inconsistent in that the energy and angular momentum content of the waveforms differs from the change in energy and angular momentum of the particle orbit which is nominally emitting the radiation. This energy inconsistency means that some of the results that have been obtained using kludged waveforms, such as signal-to-noise ratios [1], are inaccurate. The results of the semirelativistic calculations presented here allow us to estimate the magnitude of this inconsistency for inspirals into Schwarzschild black holes (see Sec. IIIC). In the future, using semirelativistic fluxes (once these are extended to spinning black holes) in the kludge in place of the post-Newtonian fluxes employed currently will yield consistent inspirals.

The key results of this paper are summarized as follows:

(1) Numerical results are presented which enable us to explore and evaluate several approximate methods of calculating energy and angular momentum fluxes from EMRI orbits, and therefore the evolution of those orbits (see Sec. III). Significantly better evolutions (compared to the results of exact Teukolskybased calculations) can be built out of the semirelativistic formalism developed here, but improved orbital evolutions can also be obtained from classic gravitational radiation estimates (Peters and Mathews) simply by choosing to work with "geodesic parameters" instead of "Keplerian parameters," with little consequence to computational cost. See Sec. III A and Fig. 3.

(2) Analytic expressions are derived for the energy $(\Delta E)$ and angular momentum $\left(\Delta L_{z}\right)$ radiated in gravitational waves for a single orbital pass near a black hole, as a function of the orbital parameters, which exactly reproduce our numerical results. See Sec. III D 2 and Eqs. (35) and (37), for the case of parabolic orbits, and Sec. 1 of the appendix for a more general discussion.

(3) Fitting functions are given which reproduce approximately, but to high accuracy, the analytic and numerical results. These functions are relatively simple expressions which could be conveniently used in place of consistently Newtonian-order expressions such as those of Peters and Mathews, which are significantly less accurate for orbits with very close periapses (see Sec. IIID 1 and Sec. 2 of the appendix). Although we present fits to the semirelativistic results only, the fitting functions have more general applicability and it should be possible to derive a fit of the same form to Teukolsky-based results once these are available for generic orbits.

The remainder of the paper will be organized as follows. In Sec. II we describe the semirelativistic scheme which we use to model the gravitational radiation from EMRIs. In Sec. III we present fluxes calculated using this approach and compare these with more accurate Teukolsky-based results, as well as with the consistently Newtonian results of Peters and Mathews. We also present analytic formulas which reproduce our numerical results, and discuss the case of hyperbolic orbits which are initially unbound but become bound to the black hole via gravitational bremsstrahlung. Finally in Sec. IV we summarize our most important results and findings. 
Throughout this paper, geometric units where $G=c=$ 1 are employed unless otherwise specified.

\section{MODEL OF GRAVITATIONAL RADIATION}

\section{A. Quadrupole approximation}

The energy and angular momentum carried away by gravitational waves from a weak-field, slow-motion source can be computed using the quadrupole formula [17]

$$
\frac{d E}{d t}=-\frac{1}{5}\left\langle\dddot{I}_{j k} \dddot{I}_{j k}\right\rangle,
$$

and

$$
\frac{d L_{z}}{d t}=-\frac{2}{5} \epsilon_{j k l}\left\langle\ddot{I}_{k a} \dddot{I}_{a l}\right\rangle,
$$

where summations are implied over repeated indices, $\boldsymbol{\epsilon}_{j k l}$ is the permutation symbol, and $I_{j k}$ is the reduced quadrupole moment of the system,

$$
I_{j k}=\int \rho\left(x_{j} x_{k}-\frac{1}{3} \delta_{j k} r^{2}\right) d^{3} x .
$$

The angle brackets \langle\rangle in (1) and (2) indicate averaging over several orbits, but parabolic trajectories (our main focus) do not have periodic orbits. Indeed, the period of a parabolic orbit is infinite, so the average energy flux over the whole orbit is zero. Therefore it is convenient to work instead in terms of $\Delta E$ and $\Delta L_{z}$, the total energy and angular momentum radiated over a single orbit, which are in general finite.

The corresponding gravitational waveform in transverse-traceless gauge is given by [17]

$$
\begin{aligned}
h_{j k}^{T T} & =\frac{2}{r}\left[P_{j l} \ddot{I}_{l m} P_{m k}-\frac{1}{2} P_{j k} P_{m l} \ddot{I}_{l m}\right], \\
P_{j k} & =\delta_{j k}-n_{j} n_{k},
\end{aligned}
$$

in which $n_{j}$ denotes the direction of propagation of the wave and $r$ is the proper distance to the source.

For orbits in the weak field, far from the black hole, the quadrupole formula applies, the source particle orbit is Keplerian, and the radiation field reduces to the Peters and Mathews result. For orbits that pass close to the black hole, the particle's geodesic orbit is no longer Keplerian and the motion is neither weak field nor slow motion, so the quadrupole formula does not describe the wave emission precisely. As described above, correcting the emission formula requires use of black hole perturbation theory (Teukolsky methods), which is computationally very challenging, but a significantly improved approximation can be obtained by using the quadrupole formula with source particle orbits modified to be a geodesic of the black hole spacetime.

To do this, first identify the Cartesian coordinates, $x_{j}$, in the quadrupole moment expression (3) with coordinates in the Schwarzschild spacetime. Treating the Schwarzschild coordinates $\{r, \theta, \phi\}$ as spherical polar coordinates, define a set of pseudo-Cartesian coordinates by

$$
x^{i}=(r \sin \theta \cos \phi, r \sin \theta \sin \phi, r \cos \theta) .
$$

In these coordinates, one can solve the Schwarzschild geodesic equations (see Sec. II B) to compute the particle orbit $x_{j}(t)$, and substitute the resulting trajectory into the quadrupole moment expression (3) to compute

$$
I^{j k}=m\left[x^{j} x^{k}-\frac{1}{3} \delta^{j k} r^{2}\right],
$$

where $m$ is the mass of the particle. Finally, we compute an estimate of the energy and angular momentum radiated using expressions (1) and (2).

This approximation for gravitational wave emission was first applied by Ruffini and Sasaki, who termed it a semirelativistic approximation [13], since it makes use of the fully relativistic orbit, but only a weak-field expression for the gravitational waves. The approach is equivalent to attaching the compact body to a string in flat space and forcing it to move on a path that corresponds to a geodesic of the Schwarzschild potential. In reality, the inspiralling body does not follow a geodesic, due to the effect of radiation reaction on the orbit. The loss of energy occurs continuously, so particle trajectories depart from a true geodesic path continuously. Instead of stable orbits, particles follow inspiralling paths with a steadily decreasing average radial distance from the center. However, in the typical case for extreme-mass-ratio inspirals, in which the rate of energy loss per orbit is small, the actual particle trajectory looks similar to a geodesic orbit for long periods, so one is justified in making an adiabatic approximation [9]; simply assume the body evolves through a sequence of geodesics and determine this sequence using the energy and angular momentum fluxes from each geodesic orbit.

The adiabatic approximation will break down when the orbital parameters change significantly on the time scale of a single orbit, which occurs only very close to the final plunge. The trajectory and waveform in this region must be computed using the computationally intensive self-force formalism (see [18] for a review).

\section{B. Geodesics}

The equations governing geodesic motion in the Schwarzschild spacetime, in the usual Schwarzschild coordinates, are given by

$$
\begin{gathered}
\left(\frac{d r}{d \tau}\right)^{2}=\left(E^{2}-1\right)+\frac{2 M}{r}\left(1+\frac{L_{z}^{2}}{r^{2}}\right)-\frac{L_{z}^{2}}{r^{2}}, \\
r^{2}\left(\frac{d \phi}{d \tau}\right)=L_{z}, \\
\left(1-\frac{2 M}{r}\right)\left(\frac{d t}{d \tau}\right)=E,
\end{gathered}
$$


where $\tau$ is the proper time along the geodesic, $L_{z}$ is the conserved specific angular momentum of the particle, $E$ is the conserved specific energy and $M$ is the mass of the central black hole. We have taken advantage of spherical symmetry to assume an equatorial orbit, $\theta=\pi / 2$, without loss of generality. The radial equation of motion (7) may be written as a cubic polynomial divided by $r^{3}$. The cubic has one, two or three roots depending on the values of $E$ and $L_{z}$. These roots correspond to turning points of the radial motion. Orbits with a single turning point plunge into the black hole and correspond to energies

$$
\begin{aligned}
& E^{2}-1>\frac{L_{z}^{2}}{r_{I}^{2}}-\frac{2 M}{r_{I}}-\frac{2 M L_{z}^{2}}{r_{I}^{3}}, \\
& \quad \text { where } r_{I}=\frac{L_{z}^{2}}{2 M}\left(1-\sqrt{1-\frac{12 M^{2}}{L_{z}^{2}}}\right) .
\end{aligned}
$$

For bound orbits, it is possible to define an orbital eccentricity by analogy with the Keplerian case. Define the position of the apoapse of the orbit to be

$$
r_{a}=\frac{1+e}{1-e} r_{p}
$$

where $r_{p}$ is the radius of the periapse. Equation (11) is used to define the eccentricity of a geodesic in terms of the turning points of the orbit $[8,10]$. This definition carries over to parabolic $\left(E^{2}=1\right)$ and hyperbolic orbits $\left(E^{2}>1\right)$. In the parabolic case, the radial geodesic equation (7) has only two turning points (the apoapse is "at infinity"), but definition (11) holds with $e=1$. In the hyperbolic case, one of the turning points has $r<0$; using this in (11) one finds $e>1$, and so in this case we call that turning point the apoapse.

For this definition for eccentricity, we use the parameters $\left(r_{p}, e\right)$ to characterize the orbit, instead of $\left(E, L_{z}\right)$. The energy and angular momentum are related to the periapse and eccentricity by

$$
\begin{gathered}
E=\sqrt{1+\frac{M(1-e)\left(4 M-(1+e) r_{p}\right)}{r_{p}\left((1+e) r_{p}-\left(3+e^{2}\right) M\right)},} \\
L_{z}=\frac{(1+e) r_{p}}{\sqrt{(1+e) \frac{r_{p}}{M}-\left(3+e^{2}\right)}} .
\end{gathered}
$$

The radial geodesic equation becomes

$$
\left(\frac{d r}{d \tau}\right)^{2}=\left(E^{2}-1\right)\left(\frac{1}{r}\right)^{3}\left(r_{a}-r\right)\left(r-r_{p}\right)\left(r-r_{-}\right)
$$

where the apoapse, $r_{a}$, and energy $E$ are given by Eqs. (11) and (12), and the third root of the potential is given by

$$
r_{-}=\frac{2(1+e) r_{p}}{\left((1+e) r_{p}-4 M\right)} \text {. }
$$

For any given eccentricity, there is a minimum value for the periapse below which the orbit plunges directly into the black hole. This occurs when the two inner turning points of the geodesic equation, $r_{-}$and $r_{p}$, coincide. A geodesic with precisely this periapse asymptotically approaches a circular orbit as it nears the periapse, and spends an infinite amount of time whirling around the black hole. The asymptotic circular orbit is an unstable orbit of the gravitational potential, and we will refer to it as the "unstable circular orbit" (UCO). The radius of the UCO determines the minimum periapse for geodesics of a fixed eccentricity. Equating $r_{-}$and $r_{p}$ yields an expression for the UCO in terms of $e$

$$
r_{\mathrm{UCO}}=\frac{2(3+e)}{1+e} M
$$

The statement that orbits with $r_{p}<r_{\mathrm{UCO}}$ are plunging is equivalent to the relationship (10) between the energy and angular momentum (see [8] for an equivalent relation in terms of the semilatus rectum). If $e=0$ the $\mathrm{UCO}$ is at the familiar innermost stable circular orbit, $r=6 \mathrm{M}$. In the extreme hyperbolic limit, $e \rightarrow \infty$, the UCO approaches the horizon $r=2 M$. Parabolic orbits $(e=1)$ have a minimal periapse of $r=4 M$. Cutler, Kennefick and Poisson [8] also discuss the UCO, but they call the line $r_{p}=r_{\mathrm{UCO}}$ the "separatrix," since it separates bound from plunging orbits in phase space.

These orbital properties can be understood by considering the radial gravitational potential $V\left(r, L_{z}\right)$, which is illustrated in Fig. 1 for a typical zoom-whirl orbit. The characteristic feature of these highly relativistic potentials is the maximum at $r_{\mathrm{UCO}}$. As an inspiral approaches plunge,

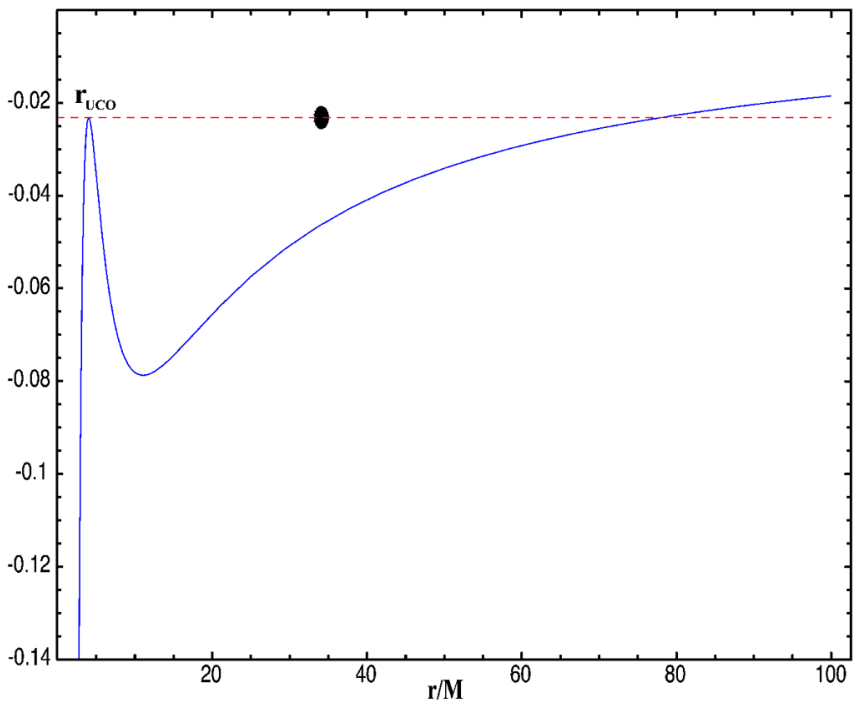

FIG. 1 (color online). Radial gravitational potential for a zoom-whirl orbit. The dashed line corresponds to the energy of the orbit. The orbit oscillates in the region where the potential (solid curve) lies below the energy line. If the energy is too high and the orbit passes inside the maximum of the radial potential $\left(r_{\mathrm{UCO}}\right)$, the particle plunges into the black hole. 
the orbit is within the potential well but close to the top of the well. That is, the periapse lies close to the UCO. The particle thus zooms out to apoapse and back, but loiters close to periapse, whirling several times around the black hole on a nearly circular orbit before zooming out to apoapse again. As one approaches the UCO, the more exaggerated the whirl phase gets and the closer the resemblance to an unstable circular orbit.

\section{Waveform structure}

The waveforms resulting from zoom-whirl orbits are easy to comprehend. During the long apoapse passage the motion of the source is relatively slow, and the ampli-
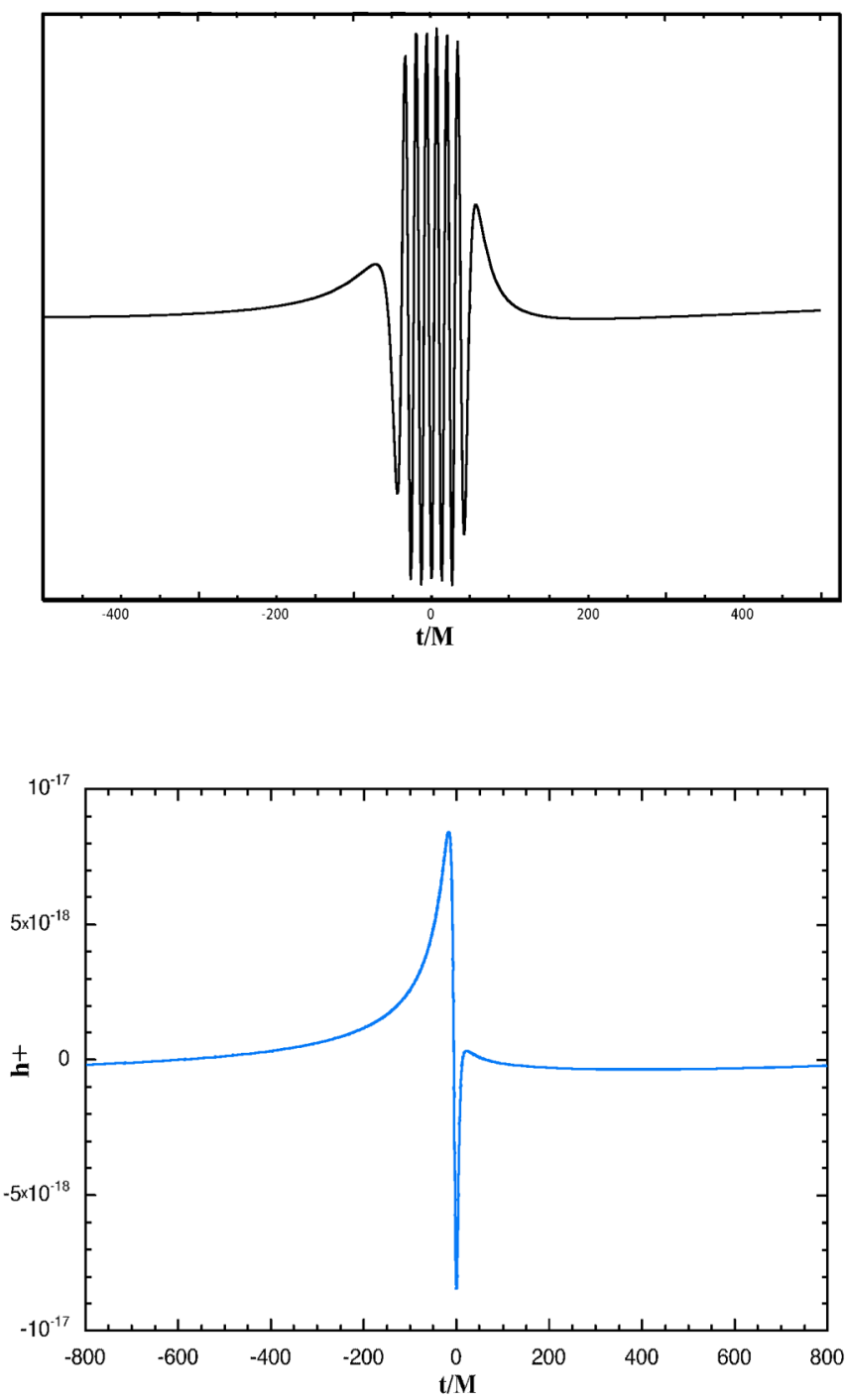

FIG. 2 (color online). Sample gravitational waveform (top) from the zoom-whirl orbit indicated in Fig. 1. We show the plus polarization of the gravitational wave as a function of time. The radiation is emitted predominantly in a high frequency burst during the whirl phase of the orbit. For comparison, the waveform from the Keplerian orbit with the same parameters is shown in the bottom diagram. tude and frequency of the gravitational wave produced are both low. Near periapse the motion is much more rapid and the signal has much higher amplitude and frequency. If the whirling phase of the orbit consists of one or more complete revolutions about the central body then the waveform will have several cycles (two for each revolution). The result is a waveform with a very low frequency (determined by the radial period of the orbital motion) and low amplitude superposed with a burst of short duration (relative to the overall period) and relatively high amplitude whose frequency is determined by the azimuthal period of the orbital motion. An example waveform is shown in Fig. 2, corresponding to the orbit indicated in Fig. 1.

Note that while the radial frequency is much too low for detection by LISA, the azimuthal $(\phi)$ frequency does fall in the LISA bandwidth for the orbits of interest. Although there is a low probability of detecting these bursts since they are too brief and infrequent (typically occurring once every few years or even longer, depending on the radial period) to have high signal-to-noise, the background of all such bursts occurring throughout our neighborhood of the Universe will create an astrophysical background of noise from which other sources must be subtracted [19].

\section{ENERGY AND ANGULAR MOMENTUM FLUXES}

The semirelativistic approximation is constructed by integrating approximate rates of energy and angular momentum flux over relativistically accurate geodesic orbits. A consistent approximation would require that the particle orbit be approximated to the same level of accuracy as employed for the fluxes. A Newtonian-order approximation, such as that of Peters and Mathews, makes use of Keplerian elliptical orbits in flat spacetime and the fluxes are of quadrupole order only. There might not appear to be much justification for using accurate orbital paths but retaining approximate fluxes. For orbits which never come close to the central black hole the semirelativistic scheme does not improve significantly on fully consistent Newtonian approximations - in nearly flat regions of spacetime all reasonable approximations fare well. However, orbits with small periapse distances are a very different case. More accurate schemes (such as those based on solution of the Teukolsky equation) show that the radiation from orbits which come close to the black hole show features that are greatly modified by the strongly curved spacetime and which are qualitatively different from those seen at large radii from the black hole. Many of these features arise from the properties of the geodesics in the strong-field regime and therefore, as argued in [14], such features can be modeled by schemes which combine exact geodesics with approximate fluxes. This approach shows significant improvements over the weak-field approximation [3], as we will see in the next section, while being 
considerably less expensive computationally than solving the Teukolsky equation.

\section{A. Comparison to Peters and Mathews and Teukolsky results}

The expressions derived in [3] for the energy and angular momentum fluxes from a Keplerian orbit are

$$
\begin{gathered}
\left\langle\frac{d E}{d t}\right\rangle=-\frac{32}{5} \frac{m}{M^{2}} \frac{(1-e)^{3 / 2}}{(1+e)^{7 / 2}}\left(1+\frac{73}{24} e^{2}+\frac{37}{96} e^{4}\right)\left(\frac{r_{p}}{M}\right)^{-5} \\
\left\langle\frac{d L_{z}}{d t}\right\rangle=-\frac{32}{5} \frac{m}{M} \frac{(1-e)^{3 / 2}}{(1+e)^{2}}\left(1+\frac{7}{8} e^{2}\right)\left(\frac{r_{p}}{M}\right)^{-7 / 2}
\end{gathered}
$$

These are Eqs. (5.4) and (5.5) of [4], but written to lowest order in the mass ratio, $m / M$, in the extreme-mass-ratio limit $M=m_{1} \gg m_{2}=m$. The eccentricity and periapse of a Keplerian orbit are given in terms of the energy and angular momentum by

$$
e^{K}=\sqrt{1-\frac{L_{z}^{2}}{M^{2}}\left(1-E^{2}\right),} \quad r_{p}^{K}=\frac{L_{z}^{2}}{M\left(1+e^{K}\right)} .
$$

To use (17) and (18) in the strong-field regime, the natural way to proceed is to evaluate the fluxes in Eqs. (17) and (18) for the Keplerian orbit with the corresponding energy and angular momentum, i.e., substitute $e^{K}$ and $r_{p}^{K}$ from (19) into (17) and (18) ("Peters and Mathews with Keplerian parameters"). This approach runs into difficulties however, since Keplerian orbits do not exist for certain valid choices of $E$ and $L_{z}$, for example, if $L_{z}^{2}>M^{2} /(1-$ $\left.E^{2}\right)$ the Keplerian eccentricity is undefined. An alternative way to proceed is to compute the geodesic eccentricity and periapse using expressions (12) and (13) and use these in the flux formulas (17) and (18), thus identifying geometrically similar orbits ("Peters and Mathews with geodesic parameters").

In Fig. 3 we compare the fluxes computed in these three ways: Peters and Mathews fluxes using Keplerian parameters, Peters and Mathews using geodesic parameters, and the semirelativistic approximation, all as a function of geodesic (relativistic) periapse for fixed geodesic (relativistic) eccentricity of $e=0.99$. For large periapse, the three approximations agree as expected, but once the periapse becomes moderate $\left(r_{p} \lesssim 50 M\right)$, the Peters and Mathews expression with Keplerian parameters begins to differ quite significantly from the other approximations. In the strongfield region $\left(r_{p} \lesssim 10 M\right)$, the semirelativistic approximation begins to differ significantly from both applications of the Peters and Mathews formula, predicting greater fluxes of both energy and angular momentum.

To verify that the semirelativistic results are an improvement over Peters and Mathews, rather than merely being different, the approximation can be compared to perturba-
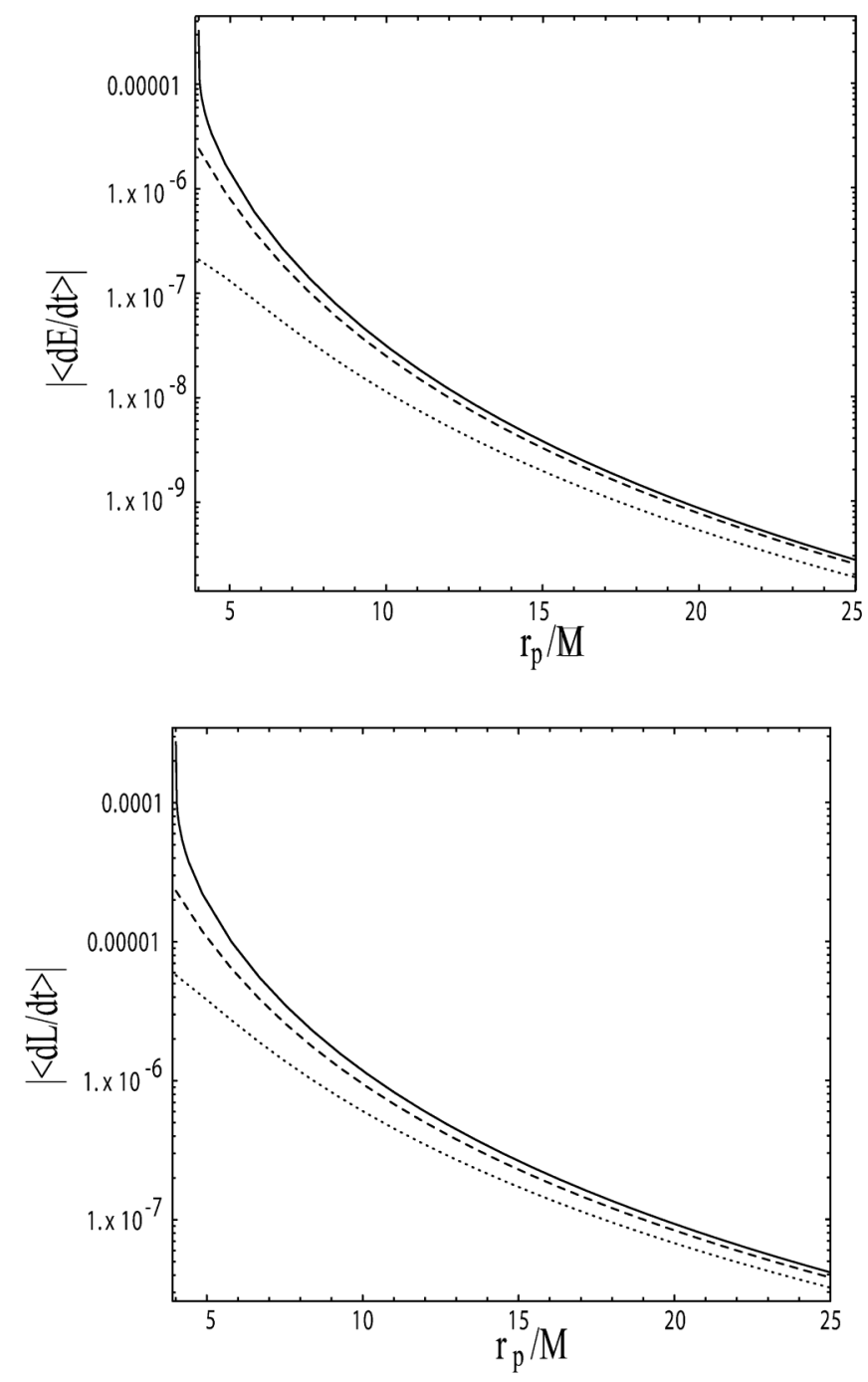

FIG. 3. Comparison between the semirelativistic results and Peters and Mathews as a function of periapse for orbits with fixed geodesic eccentricity $e=0.99$. The solid lines are the results from the semirelativistic approximation discussed here. The dashed and dotted lines are the Peters and Mathews results with geodesic parameters and Keplerian parameters, respectively. We use a logarithmic vertical scale and plot the absolute value of the energy (upper plot) and angular momentum (lower plot) fluxes.

tive results from integration of the Teukolsky equation. Very few results are available for high eccentricities in the Teukolsky formalism, so the comparisons here are shown at lower eccentricities. In Fig. 4, the semirelativistic and Peters and Mathews fluxes are compared to Teukolsky calculations [10] for orbits with eccentricity $e=0.5$ and a variety of periapses. As one would expect, the semirelativistic approximation is not superior to a consistent Newtonian approach for periapses greater than about $\sim 50 M$ (sometimes it does worse and sometimes better than the Peters and Mathews results, but never extremely different). For periapses less than $\sim 50 M$, the semirelativ- 
istic method improves significantly upon the consistent Peters and Mathews approximation. The improvement gained using the semirelativistic approximation was also noted by [20] in comparisons to a selection of Teukolskybased results. Thus for the type of orbit of interest to this paper (highly eccentric orbits with close periapses), consistent Newtonian approximations should be regarded with suspicion, but approximations which make use of exact geodesics (like the semirelativistic approximation), will fare very well qualitatively and quite well quantitatively, as long as the periapse is not extremely small.
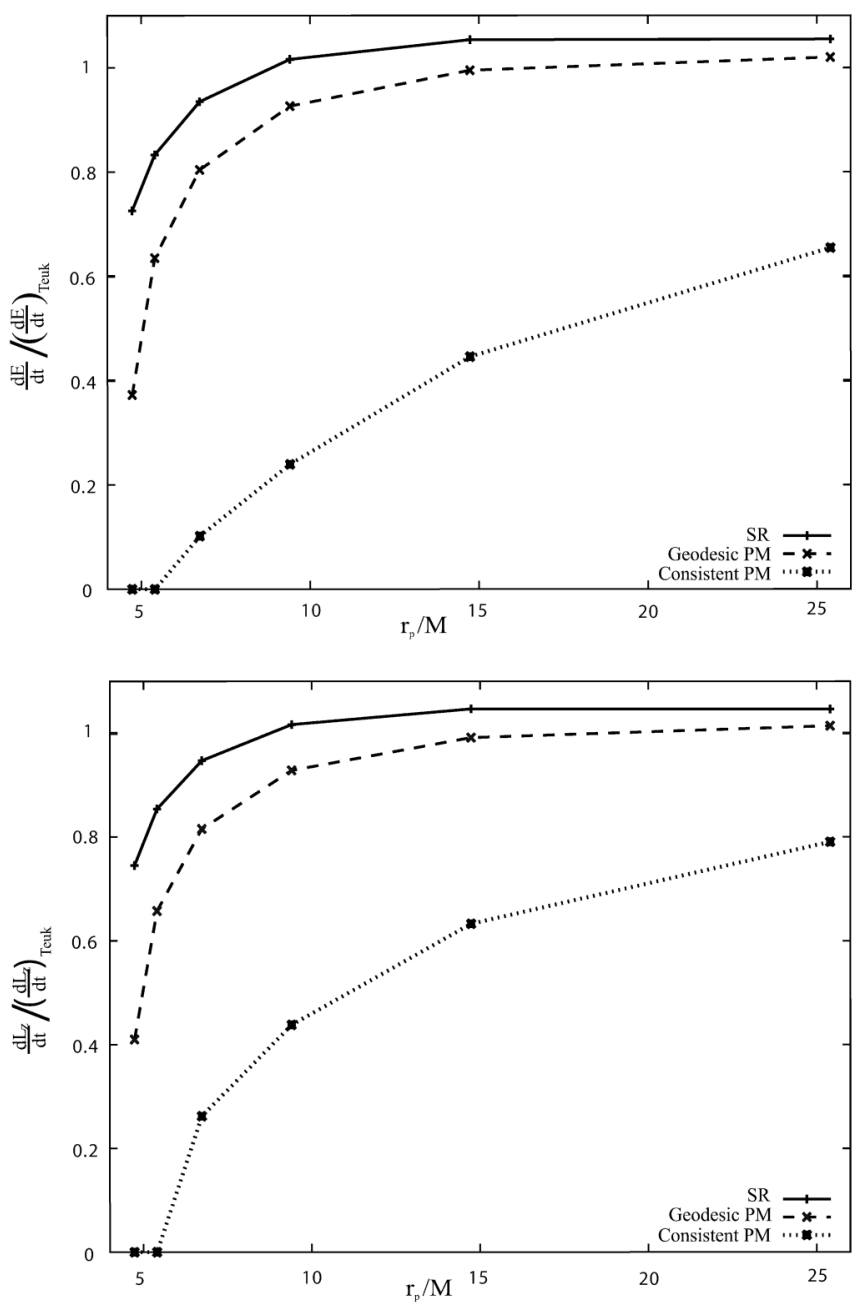

FIG. 4. Comparison between accurate Teukolsky results and various approximations, for orbits with fixed eccentricity $e=$ 0.5 and a variety of periapses. The plots show the ratio of the flux computed using a given approximation to the flux obtained from the Teukolsky calculation. The upper plot shows the ratio of the energy fluxes, while the lower plot is the ratio of the angular momentum fluxes. In both plots, the solid lines are the semirelativistic results. The dashed and dotted lines are for Peters and Mathews, evaluated with geodesic parameters and Keplerian parameters, respectively. The latter lines cut off at small periapse since there are no corresponding Keplerian orbits in that region.
What is perhaps more surprising is that one may obtain an improved approximation from the Newtonian-order expressions (i.e., Peters and Mathews) if one carefully chooses the Newtonian parameters which are to be equated with the "true" curved space parameters (i.e., geodesic parameters rather than Keplerian parameters). While the semirelativistic approximation is always an improvement over this in the strong-field regime, the gain is only significant for very close periapses, $r_{p} \lesssim 10 M$. In fact, for small eccentricities there is no significant gain using the semirelativistic fluxes. For a circular orbit of radius $r_{0}$, the quadrupole formulas (1) and (2) tell us that $\langle d E / d t\rangle=$ $-32 r_{0}^{4} \Omega_{\phi}^{6}$ and $\left\langle d L_{z} / d t\right\rangle=-32 r_{0}^{4} \Omega_{\phi}^{5}$, where $\Omega_{\phi}=$ $d \phi / d t$ is the angular velocity. For both a Keplerian orbit and a circular geodesic of the Schwarzschild metric, $\Omega_{\phi}=$ $1 / r_{0}^{3 / 2}$. Therefore, the standard Peters and Mathews result is recovered exactly for circular orbits using either geodesic or Keplerian parameters.

We are primarily interested in highly eccentric orbits, for which the semirelativistic results are a significant improvement over any method based on Peters and Mathews. Nonetheless, if one does not wish the additional computational burden of evaluating more accurate semirelativistic flux expressions, a significant improvement can still be gained by evaluating the Newtonian fluxes using geodesic parameters.

\section{B. Phase space structure}

An inspiral sequence may be constructed from the semirelativistic fluxes by integrating $\left(d E / d t, d L_{z} / d t\right)$. While the duration of the inspiral depends on the value of $d E / d t$, the sequence of geodesics that the inspiral passes through in the $\left(E, L_{z}\right)$ phase space depends only on the ratio $d E / d L_{z}$. Equivalently, in the $\left(r_{p}, e\right)$ phase space, it depends only on

$$
\frac{d r_{p}}{d e}=\frac{\frac{\partial L}{\partial e} \frac{d E}{d L_{z}}-\frac{\partial E}{\partial e}}{\frac{\partial E}{\partial r_{p}}-\frac{\partial L_{z}}{\partial r_{p}} \frac{d E}{d L_{z}}} .
$$

It turns out that the semirelativistic approximation reproduces the ratio $d E / d L_{z}$ to a very high accuracy when compared to the Teukolsky results. While the value of $\Delta E$ can differ by as much as $25 \%$, the ratio $d E / d L_{z}$ is recovered to better than $5 \%$. This means that the structure of the semirelativistic phase space will be a good approximation to the true structure, although there is some error in the estimated duration of inspirals.

This is an interesting result from the point of view of detection of EMRIs with LISA. An error in the time scale of an inspiral can be partially corrected by a change in the mass ratio. Since the phase space trajectory is well approximated, an inspiral waveform computed using this approach might be a moderately good fit to a true inspiral waveform with a slightly different mass ratio, and therefore 
could be used as a detection template over sufficiently short time stretches. This may not be practical, since the error in $d E / d t$ is not a constant factor, which would require varying the mass ratio over the inspiral. Moreover, other features that these approximations do not include (such as the "conservative" part of the self-force) may lead to rapid dephasing of the kludge templates. This is nonetheless an interesting result.

The accuracy with which the phase space structure is reproduced can be understood by considering what happens at the extremes of an inspiral. When the periapse is very large, the semirelativistic approximation is good, and is expected to reproduce $d E / d L_{z}$ accurately. For an orbit that sits on the separatrix between plunging and nonplunging orbits $\left(r_{p} \sim r_{\mathrm{UCO}}\right)$, the geodesic spends an infinite amount of time whirling about the black hole on a nearly circular orbit at the UCO. The flux of energy and angular momentum is totally dominated by the circular part of the orbit. For any radiation field in a spherically symmetric spacetime, the energy and angular momentum fluxes carried away from a circular orbit obey the relation $d E / d t=$ $\Omega_{\phi}\left(d L_{z} / d t\right)$, where $\Omega_{\phi}$ is the angular velocity on that orbit [8]. The quadrupole formulas (1) and (2) reproduce this result for a circular orbit. Since we use the correct input geodesic, the semirelativistic approximation should and does give $d E / d L_{z}$ accurately on the separatrix. Since we have the correct result in both extremes, it is perhaps less surprising that we also do quite well at points in between.

Figure 5 illustrates some inspiral trajectories in the $\left(r_{p}, e\right)$ plane. The trajectory properties are determined largely by two curves - the separatrix where the inspiral

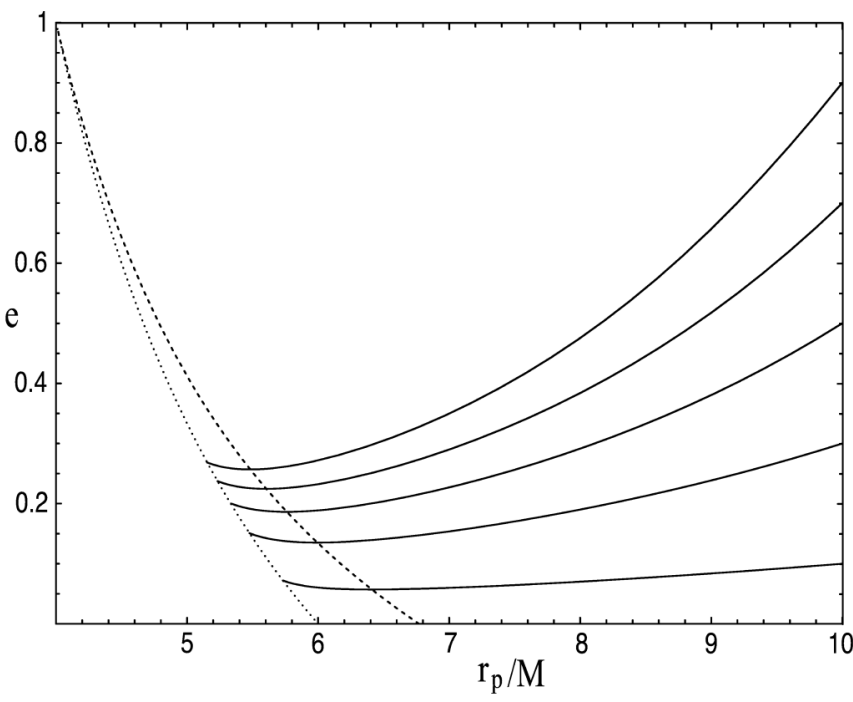

FIG. 5. Sample inspiral trajectories in the $\left(r_{p}, e\right)$ plane. The solid lines illustrate inspiral trajectories. The dotted line marks the separatrix and all points to the left of this line are plunging orbits. The dashed line is the locus of points with $d e / d t=0$. In the region between this line and the separatrix, $d e / d t>0$ on all trajectories. ends as the particle plunges into the black hole and the locus $d e / d t=0$. These are both marked on Fig. 5. In general an inspiral will begin with high eccentricity and moderate periapse. Both the periapse and eccentricity initially decrease, and this evolution continues until the trajectory intersects the $d e / d t=0$ curve. After that point, the periapse continues to decrease, but the eccentricity increases until the trajectory reaches the separatrix and the particle plunges. As expected from previous arguments, these general properties are in good agreement with results based on Teukolsky calculations [8] and quite different to Peters and Mathews inspirals (which, for instance, have monotonically decreasing eccentricity). The location of the separatrix is a property of the geodesics, and is therefore precisely reproduced in this approximation. The $d e / d t=$ 0 curve depends on the expression used for the energy and angular momentum fluxes and is different here, but only slightly. In this approximation, the $d e / d t=0$ curve intersects the $e=0$ axis at $r_{p}=6.770 M$, compared to $r_{p}=$ $6.681 M$ in the Teukolsky case [8].

The increase in eccentricity prior to plunge is a generic feature of EMRIs, but it is as much a property of the radial potential as it is of the flux model. As discussed earlier, realistic gravitational waves will give $\dot{E}=\Omega_{\phi} \dot{L}_{z}=((1+$ $e) /(2(3+e)))^{3 / 2} \dot{L}_{z}$ on the separatrix. The leading order piece in both the numerator and denominator of Eq. (20) thus vanishes in the limit $r_{p} \rightarrow r_{\mathrm{UCO}}(e)$, but the leading correction to both is from the logarithmic piece of $d E / d L_{z}$, and hence we find $d r_{p} / d e<0$. However, this conclusion still holds if the fluxes do not satisfy the circular orbit condition and the cancellations do not occur. The coordinate derivatives are such that, independent of the value of $d E / d L_{z}$ on the separatrix,

$$
\frac{d r_{p}}{d e} \approx-\frac{4(3+e)}{(1-e)(1+e)^{2}} .
$$

The nature of the potential thus forces either $r_{p}$ or $e$ to increase in the approach to plunge.

A final point to note from Fig. 5 is that $\dot{e} \propto e$ near $e=0$. This property of the inspirals means that an initially eccentric orbit cannot circularize in this model, although the eccentricity at plunge can be arbitrarily small. The property once again derives from the circular condition $\dot{E}=$ $\Omega_{\phi} \dot{L_{z}}$, which ensures that circular orbits remain circular under radiation reaction. This is discussed in more detail in [15], where corrections are given to enforce this relation in kludged inspirals. In the semirelativistic waveform model, the condition is automatically satisfied and no correction is required.

\section{C. "Kludge" waveform inconsistency}

As mentioned in the introduction, waveforms based on the semirelativistic approximation are being used extensively to scope out LISA data analysis [14-16]. The wave- 
forms are generated by first constructing an inspiral trajectory and then using the semirelativistic construction of the quadrupole moment tensor to compute a waveform. In the most basic form of this kludge [14], the phase space trajectory for inspirals into nonspinning black holes is computed by integrating the Peters and Mathews energy and angular momentum fluxes (17) and (18). This leads to an inconsistency since the energy and angular momentum content of the gravitational waves differs from the energy and angular momentum lost by the inspiralling particle that is nominally generating the radiation. We can estimate this inconsistency using the semirelativistic results. A phase space trajectory is generated using the fluxes (17) and (18) and Eq. (20). We choose to specify the eccentricity of the inspiral at plunge and integrate backwards along the inspiral trajectory. By integrating the semirelativistic fluxes along this trajectory, we calculate the total energy and angular momentum content of the gravitational waves. Figure 6 shows the ratio of the gravitational wave energy flux to the change in energy of the particle orbit as a function of the time until plunge (in units of $M^{2} / \mathrm{m}$ ). Time along the inspiral trajectory therefore decreases toward the right. There is a curve for each eccentricity at plunge from $e=0.1$ to $e=0.9$ in intervals of 0.1 . We see that there is a significant inconsistency in the kludge waveforms. For low eccentricity at plunge, the kludge gravitational waves contain less energy than they should, but for eccentricity at plunge greater than about 0.25 , they contain too much energy, as much as a factor of 3 in extreme cases. This means that signal-to-noise ratios (SNRs) computed from these waveforms are likely to be overestimates of the true SNRs. It is not clear from these results whether this discrepancy will be larger or smaller when the central black hole is spinning, but this will be investigated in the future [16]. However, it is important to be aware of the existence and magnitude of this problem when interpreting

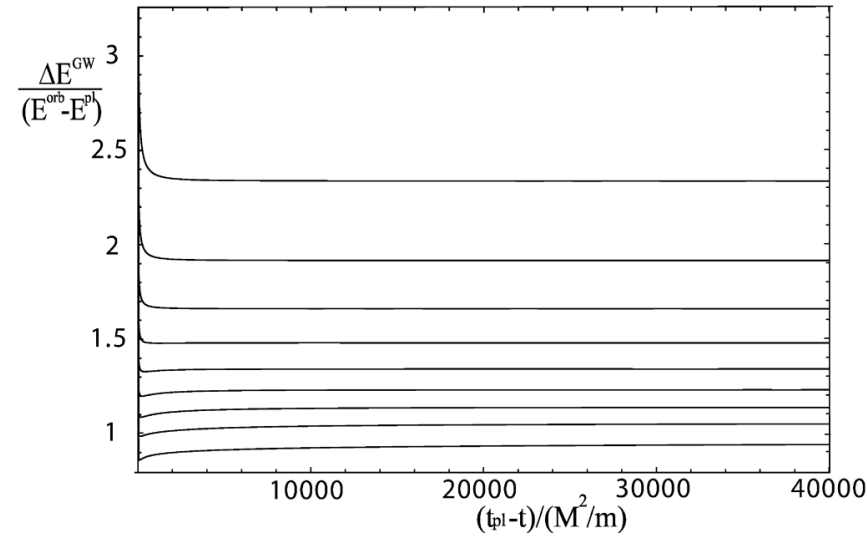

FIG. 6. Ratio of the energy content of kludge gravitational waves to the change in energy of the source particle orbit, relative to the energy at plunge. This is shown as a function of time until plunge (in unite of $M^{2} / m$ ) for eccentricities at plunge $e_{\mathrm{pl}}=0.1,0.2 \ldots 0.9$ (from lowermost curve to uppermost). results based on the kludge waveforms. If semirelativistic fluxes were used to integrate the phase space trajectories, there would be no such inconsistency and this might therefore be another future application of these results, once they are extended to spinning black holes.

\section{Analytic results}

The previous results have shown the usefulness of the semirelativistic approximation, but the described method, based on integration of the geodesic equations, is not easy to implement in numerical simulations. In this section, we present some analytic results based on the semirelativistic approximation which can be easily evaluated without numerical integration of orbits.

\section{Fitting functions for $\Delta E$ and $\Delta L_{z}$}

A useful tool for simulations is a fitting function that has a simple form and which reproduces the semirelativistic results for $\Delta E$ and $\Delta L_{z}$ to reasonable accuracy. For a geodesic of given eccentricity, the periapse can have any value between the UCO for that eccentricity (16) and infinity. For large values of the periapse, the orbit is entirely within the weak-field region of the spacetime. The orbit and radiation will therefore look very like those from a Keplerian orbit, as described by expressions (17) and (18) [3]. Multiplying these expressions by the Keplerian orbital period, the energy and specific angular momentum lost on a single pass may be found to be

$$
\begin{gathered}
\Delta E=-\frac{64 \pi}{5} \frac{m}{M} \frac{1}{(1+e)^{7 / 2}}\left(1+\frac{73}{24} e^{2}+\frac{37}{96} e^{4}\right)\left(\frac{r_{p}}{M}\right)^{-7 / 2} \\
\Delta L_{z}=-\frac{64 \pi}{5} m \frac{1}{(1+e)^{2}}\left(1+\frac{7}{8} e^{2}\right)\left(\frac{r_{p}}{M}\right)^{-2} \cdot
\end{gathered}
$$

In the parabolic case $(e=1)$, these become

$$
\begin{gathered}
\Delta E=-\frac{85 \pi}{12 \sqrt{2}} \frac{m}{M}\left(\frac{r_{p}}{M}\right)^{-7 / 2} \\
\Delta L_{z}=-6 \pi m\left(\frac{r_{p}}{M}\right)^{-2} .
\end{gathered}
$$

As the periapse approaches the UCO, the energy and angular momentum lost per pass increases. In fact, ignoring radiation reaction, the energy and angular momentum losses diverge as $r_{p} \rightarrow r_{\mathrm{UCO}}$. This is because the geodesic with $r_{p}=r_{\mathrm{UCO}}$ spends an infinite amount of time whirling around the black hole with $r \approx r_{\mathrm{UCO}}$. In practice, radiation reaction will prevent this situation arising (for a discussion of the transition from inspiral to plunging orbits see [21]). However, the energy and angular momentum lost should increase rapidly as the periapse approaches $r_{\mathrm{UCO}}$, since the orbit whirls around the black hole an increasing number of times. 
As discussed previously, the whirl behavior is a property of the geodesics. Thus, although the semirelativistic treatment of the radiation is only approximate, one will still see this divergent behavior as $r_{p} \rightarrow r_{\mathrm{UCO}}$, since the source for the radiation is an exact geodesic trajectory. This feature is missing in the Keplerian treatment [3]. For an extreme zoom and whirl orbit, most of the gravitational radiation is emitted during the whirl phase, when the particle is on an approximately circular orbit. It is reasonable to guess that the total losses due to gravitational radiation are roughly proportional to the number of times the particle whirls around the black hole.

In the parabolic case, one can estimate the number of whirls by computing the proper time taken for the orbit to pass from periapse to some "whirling radius," $r_{w}$. This is found using (14) to be

$$
\tau=\frac{1}{\sqrt{2 M}} \int_{r_{p}}^{r_{w}} \frac{r^{3 / 2}}{\sqrt{\left(r-r_{p}\right)\left(r-\frac{2 M r_{p}}{r_{p}-2 M}\right)}} d r
$$

The radius does not change significantly during the whirl phase, so approximating the numerator by $r_{p}^{3 / 2}$

$\tau \approx \frac{1}{\sqrt{2 M}} r_{p}^{3 / 2} \cosh ^{-1}\left(\frac{2\left(r_{p}-2 M\right)}{r_{p}\left(r_{p}-4 M\right)}\left[r_{w}-\frac{r_{p}^{2}}{2\left(r_{p}-2 M\right)}\right]\right)$.

Using the same assumption, $d \phi / d \tau \approx L / r_{p}^{2}$ and we estimate that while $r<r_{w}$, the number of azimuthal cycles that the particle completes is

$$
\begin{aligned}
N_{\text {whirls }}= & \frac{\Delta \phi}{2 \pi} \\
\approx & \sqrt{\frac{r_{p}}{r_{p}-2 M} \frac{1}{\pi} \cosh ^{-1}\left(\frac{2\left(r_{p}-2 M\right)}{r_{p}\left(r_{p}-4 M\right)}\right.} \\
& \left.\times\left[r_{w}-\frac{r_{p}^{2}}{2\left(r_{p}-2 M\right)}\right]\right) .
\end{aligned}
$$

The radius $r_{w}$ should be chosen to define the start and end of the whirl phase. Our objective is to guess a functional form that approximates the energy and angular momentum loss when $r_{p} \approx r_{\mathrm{UCO}}$ and we assume that $d E$ and $d L_{z}$ are proportional to (28) in that limit. This is likely to be a particularly good approximation for highly eccentric orbits, in which the "zoom" and "whirl" phases are quite distinct. Appropriate fitting functions should approach (22) and (23) in the limit $r_{p} \rightarrow \infty$ and should diverge like (28) as $r_{p} \rightarrow r_{\mathrm{UCO}}$. Working once again in the parabolic case, the simplest such function is

$$
\begin{aligned}
\frac{M}{m} \Delta E= & A^{E} \cosh ^{-1}\left[1+B^{E}\left(\frac{4 M}{r_{p}}\right)^{6} \frac{M}{r_{p}-4 M}\right] \\
& +C^{E}\left(\frac{r_{p}}{M}-4\right)\left(\frac{M}{r_{p}}\right)^{9 / 2} .
\end{aligned}
$$

One could fix all three coefficients by matching the behavior in the limit $r_{p} \rightarrow r_{\mathrm{UCO}}$, but (29) will not then necessarily reproduce the asymptotic form (24). Instead, we fix $A^{E}$ and $B^{E}$ using an expansion near $r_{p}=r_{\mathrm{UCO}}$ and then fix $C^{E}=-\left(85 \pi /(12 \sqrt{2})+64 A^{E} \sqrt{2 B^{E}}\right)$ to match asymptotically.

The next section will demonstrate how exact expressions for the energy and angular momentum radiated in our model may be obtained in terms of elliptic integrals. Using these full analytic expressions, we can predict the values of the fitting function coefficients

$$
\begin{gathered}
A^{E}=-\frac{\sqrt{2}}{10}=-0.141421, \quad B^{E}=0.752091, \\
C^{E}=-4.634643 .
\end{gathered}
$$

The equivalent fitting function for the angular momentum lost is

$$
\begin{aligned}
\frac{\Delta L_{z}}{m}= & A^{L_{z}} \cosh ^{-1}\left[1+B^{L_{z}}\left(\frac{4 M}{r_{p}}\right)^{3} \frac{M}{r_{p}-4 M}\right] \\
& +C^{L_{z}}\left(\frac{r_{p}}{M}-4\right)\left(\frac{M}{r_{p}}\right)^{3}
\end{aligned}
$$

with coefficients

$$
\begin{gathered}
A^{L_{z}}=-\frac{4 \sqrt{2}}{5}=-1.13137, \quad B^{L_{z}}=1.31899, \\
C^{L_{z}}=-\left(6 \pi+8 A^{L_{z}} \sqrt{2 B^{L_{z}}}\right)=-4.149103 .
\end{gathered}
$$

The fitting function (29) can be used to match the lowest order terms in an expansion of $\Delta E$ near $r_{p}=r_{\mathrm{UCO}}$ and $r_{p} \rightarrow \infty$. It is possible to add additional terms to give a more general function which can match $\Delta E$ at arbitrary orders

$$
\begin{aligned}
\frac{M}{m} \Delta E= & \left(\sum_{n=0}^{N} A_{n}^{E}\left(\frac{M\left(r_{p}-4 M\right)}{r_{p}^{2}}\right)^{n}\right) \cosh ^{-1}\left[1+B_{0}^{E}\left(\frac{4 M}{r_{p}}\right)^{N_{E}-1} \frac{M}{r_{p}-4 M}\right]+\frac{M^{N_{E} / 2}\left(r_{p}-4 M\right)}{r_{p}^{1+\left(N_{E} / 2\right)}} \sum_{n=0}^{N} C_{n}^{E}\left(\frac{M\left(r_{p}-4 M\right)}{r_{p}^{2}}\right)^{n} \\
& +\frac{M^{1+\left(N_{E} / 2\right)}\left(r_{p}-4 M\right)}{r_{p}^{2+\left(N_{E} / 2\right)}} \sum_{n=0}^{N-1} B_{n+1}^{E}\left(\frac{M\left(r_{p}-4 M\right)}{r_{p}^{2}}\right)^{n} .
\end{aligned}
$$


In this, we fix $N_{E}=7$ to give the correct leading order behavior (24) as $r_{p} \rightarrow \infty$. The parameter $N$ indicates the order of the fit, i.e., the number of terms we include. The second and third series have terms in common, but writing the expansion in this way allows one to read off consecutive coefficients easily. The next section will show that expanding $\Delta E$ about the separatrix gives terms in $\left(r_{p}-\right.$ $4 M)^{j} \ln \left(r_{p}-4 M\right)$ and in $\left(r_{p}-4 M\right)^{k}$, while an expansion as $r_{p} \rightarrow \infty$ gives terms of the form $1 / r_{p}^{\left(N_{E} / 2\right)+l}$. The coefficient of the $j=0$ term gives $A_{0}^{E}$, then the $k=0$ term gives $B_{0}^{E}$ and the $l=0$ term gives $C_{0}^{E}$. Continuing in this way, the $j, k, l=n$ terms determine $A_{n}^{E}, B_{n}^{E}$ and $C_{n}^{E}$ respectively. Thus, an expansion to order $N$ will match the lowest $N+1$ terms in $j, k$ and $l$. A similar fitting form may be used for $\Delta L_{z} / M$, but with $N_{E}$ replaced by $N_{L_{z}}=4$ [once again, to reproduce the correct leading order behavior (25) as $r_{p} \rightarrow \infty$ ]. Figure 7 illustrates how the fitting
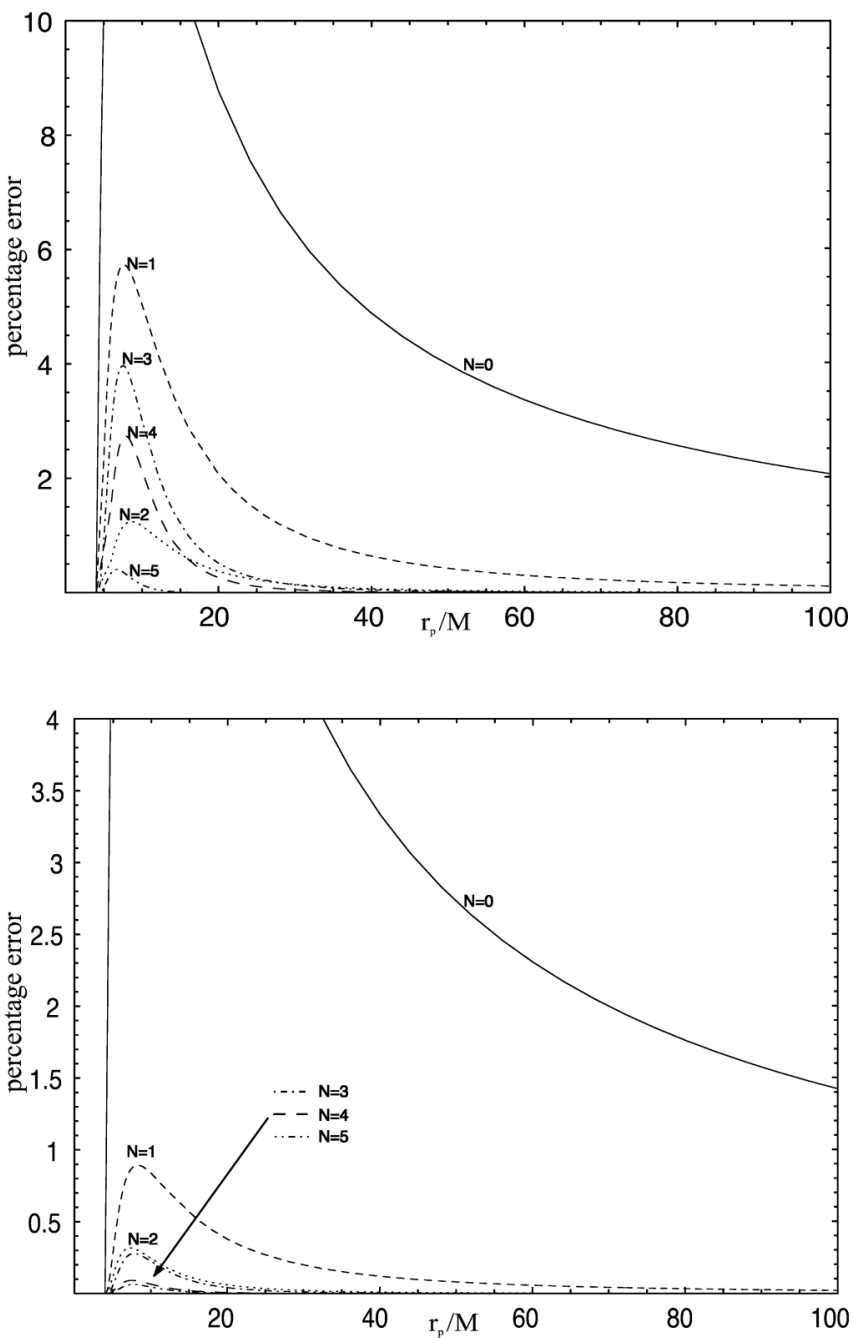

FIG. 7. Error using fitting functions to approximate the analytic expressions for the energy (upper plot) and angular momentum loss (lower plot). In each plot, the absolute percentage error in the fit is shown for fitting functions of various orders, $N=2, \cdots, 6$. functions converge as the order of the fit, $N$, is increased. We see that as $N$ increases, the fit improves at large radii, but initially gets slightly worse at moderate radii before converging there also. The $N=2$ fit is accurate to about $1 \%$ everywhere, so we include these parameters here

$$
\begin{gathered}
A_{0}^{E}=-0.141421, \quad A_{1}^{E}=0, \quad A_{2}^{E}=-1.20797, \\
B_{0}^{E}=0.752091, \quad B_{1}^{E}=-103.215 \\
B_{2}^{E}=727.515, \quad C_{0}^{E}=-4.63464 \\
C_{1}^{E}=69.1683, \quad C_{2}^{E}=-439.378 \\
A_{0}^{L_{z}}=-1.13137, \quad A_{1}^{L_{z}}=0, \quad A_{2}^{L_{z}}=0, \\
B_{0}^{L_{z}}=1.31899, \quad B_{1}^{L_{z}}=-53.4491 \\
B_{2}^{L_{z}}=29.7857, \quad C_{0}^{L_{z}}=-4.1491 \\
C_{1}^{L_{z}}=25.4129, \quad C_{2}^{L_{z}}=15.1726
\end{gathered}
$$

This fitting function was derived using simple arguments about how the energy and angular momentum lost behave. These arguments are valid in general for radiation that is produced by a body orbiting in the Schwarzschild potential and will apply to fluxes computed using the Teukolsky formalism. In a separate paper [22] we derive a fit of this form to Teukolsky data computed for parabolic orbits in [23], which even for $N=2$ is accurate to $<0.2 \%$ everywhere.

This simple fitting function is clearly a useful and accurate way to evolve EMRI orbits. In the case of arbitrary eccentricity, a similar type of fitting function can be derived, but the coefficients $A_{0}^{E}$ etc. are now functions of eccentricity. In the semirelativistic approximation, the functions can be evaluated explicitly. This is discussed in more detail in Sec. 2 of the appendix. It is more complicated to compute a fit to Teukolsky-based fluxes, since the coefficients in the expansion must be further expanded as functions of eccentricity. However, it should be possible to derive a reasonable fit using a polynomial ansatz, of the form suggested by the semirelativistic results. Once sufficient Teukolsky-based data are available, this fitting procedure will allow us to generate a comparatively simple analytic expression for use in computation of EMRIs.

\section{Exact expression}

As mentioned in the preceding section, it is possible to derive exact analytic expressions for the radiation loss predicted by our quadrupole approximation. This is possible because in any axisymmetric spacetime, the rate of energy loss at a given moment in time cannot depend on the absolute value of the $\phi$ coordinate of the particle, since a shift $\phi \rightarrow \phi+\phi_{0}$ will leave the spacetime unchanged (note that the energy flux in a given direction will be dependent on the relative difference in $\phi$ between the source and the observer). In the quadrupole approximation used here to compute the gravitational radiation, $d E / d t$ is 
given by the square of the third time derivative of the quadrupole moment tensor. This is a function only of the particle coordinates $r(t)$ and $\phi(t)$. By the axisymmetry argument, the expression for $d E / d t$ can depend only on $r, \dot{r}, \ddot{r}, \dddot{r}, \dot{\phi}, \ddot{\phi}$ and $\dddot{\phi}$. For geodesics of the Schwarzschild spacetime (also for equatorial orbits in the Kerr spacetime), $d \phi / d \tau$ and $d t / d \tau$ are rational functions of $r$ only and $(d r / d \tau)^{2}=V(r)$ is a cubic or quartic polynomial in $r$. Any derived expression, such as $d E / d r=$ $(d E / d t) /(d r / d t)$, will therefore be a rational function of polynomials in $r$ and $\sqrt{V(r)}$. It is known [24] that the integral of any rational function of polynomials in $x$ and $y$, where $y^{2}$ is a cubic or quartic polynomial in $x$, can be expressed in terms of elliptic integrals. One can therefore write the energy and angular momentum radiated in closed form in terms of elliptic integrals.

By substitution of the geodesic equations (8), (9), and (14) into (1)-(3), we may write $d E / d t$ and $d L_{z} / d t$ as functions of $r$ and then integrate over one orbit. In the parabolic case, the energy loss is found to be

$$
\begin{aligned}
\frac{M}{m} \Delta E= & -\frac{8 \sqrt{2} M^{21 / 2}}{1673196525\left(r_{p}-2 M\right)^{2} r_{p}^{17 / 2}} \\
& \times\left[\mathbf{E}\left(\sqrt{\frac{2 M}{r_{p}-2 M}}\right) f_{1}\left(\frac{r_{p}}{M}\right)\right. \\
& \left.+\mathbf{K}\left(\sqrt{\frac{2 M}{r_{p}-2 M}}\right) f_{1}\left(\frac{r_{p}}{M}\right)\right]
\end{aligned}
$$

where

$$
\begin{aligned}
f_{1}(y)= & -2 y(27850061568-83550184704 y \\
& +117662445984 y^{2}-102686941680 y^{3} \\
& +64808064704 y^{4}-33026468872 y^{5} \\
& +12784148218 y^{6}-2873196259 y^{7} \\
& +185808888 y^{8}+17119626 y^{9}+2451526 y^{10} \\
& \left.+368640 y^{11}+20480 y^{12}\right)
\end{aligned}
$$

and

$$
\begin{aligned}
f_{2}(y)= & (-72901570560+274404834816 y \\
& -424693524096 y^{2}+378109481088 y^{3} \\
& -249480499840 y^{4}+154011967968 y^{5} \\
& -84437171728 y^{6}+31689370996 y^{7} \\
& -6231594434 y^{8}+321950817 y^{9} \\
& +27462280 y^{10}+4073612 y^{11}+696320 y^{12} \\
& \left.+40960 y^{13}\right) .
\end{aligned}
$$

In this, $\mathbf{K}$ and $\mathbf{E}$ are the complete elliptic integrals of the first and second kinds, respectively, defined by $[24,25]$

$$
\begin{aligned}
& \mathbf{K}(k)=\int_{0}^{\pi / 2} \frac{d \phi}{\sqrt{1-k^{2} \sin ^{2} \phi}}, \\
& \mathbf{E}(k)=\int_{0}^{\pi / 2} \sqrt{1-k^{2} \sin ^{2} \phi} d \phi .
\end{aligned}
$$

The corresponding result for the angular momentum lost is

$$
\begin{aligned}
\frac{\Delta L_{z}}{m}= & \frac{64 M^{7}}{24249225 r_{p}^{11 / 2}\left(r_{p}-2 M\right)^{3 / 2}} \\
& \times\left[\mathbf{E}\left(\sqrt{\frac{2 M}{r_{p}-2 M}}\right) g_{1}\left(\frac{r_{p}}{M}\right)\right. \\
& \left.+\mathbf{K}\left(\sqrt{\frac{2 M}{r_{p}-2 M}}\right) g_{2}\left(\frac{r_{p}}{M}\right)\right]
\end{aligned}
$$

where

$$
\begin{aligned}
g_{1}(y)= & y\left(181817664-363635328 y+245236248 y^{2}\right. \\
& -49673460 y^{3}-7833906 y^{4}+2016105 y^{5} \\
& \left.+283252 y^{6}+35896 y^{7}+4120 y^{8}\right)
\end{aligned}
$$

and

$$
\begin{aligned}
g_{2}(y)= & \left(71285760-324389184 y-468548880 y^{2}\right. \\
& -277856496 y^{3}+54521424 y^{4}+6181872 y^{5} \\
& -1630457 y^{6}-238086 y^{7}-31776 y^{8} \\
& \left.-4120 y^{9}\right) .
\end{aligned}
$$

These exact expressions can be used to derive the fitting function described in the previous section. As $r_{p} \rightarrow \infty$, the argument of the elliptic integrals, $\sqrt{2 M /\left(r_{p}-2 M\right)} \rightarrow 0$. In a series expansion of the integrals about $k=0$ the lowest five orders in $k$ cancel and one successfully recovers (24) and (25).

As $r \rightarrow r_{\mathrm{UCO}}=4 M$, the argument of the elliptic integrals $\sqrt{2 M /\left(r_{p}-2 M\right)} \rightarrow 1$ and the elliptic integrals diverge. Using [24] and some algebraic manipulation, the asymptotic forms of the elliptic integrals as $k \rightarrow 1$ are found to be

$$
\begin{aligned}
\mathbf{K}(k)= & -\frac{1}{2} \ln \left(1-k^{2}\right)+2 \ln 2-\frac{1}{8}\left(1-k^{2}\right) \ln \left(1-k^{2}\right) \\
& +O\left(1-k^{2}\right) \\
\mathbf{E}(k)= & 1-\frac{1}{4}\left(1-k^{2}\right) \ln \left(1-k^{2}\right)+\left(\ln 2-\frac{1}{4}\right)\left(1-k^{2}\right) \\
& +O\left(\left(1-k^{2}\right)^{2} \ln \left(1-k^{2}\right)\right) .
\end{aligned}
$$

The asymptotic form of (35)-(37) as $r_{p} \rightarrow 4 M$ is

$$
\frac{d X}{\eta} \approx p_{X} \ln \left(\frac{r_{p}}{M}-4\right)+q_{X}+O\left(\frac{r_{p}}{M}-4\right) .
$$

In this, $X$ refers to either $E$ or $L_{z} / M$. The values of the constants are 


$$
\begin{aligned}
& p_{E}=\frac{1}{5 \sqrt{2}}, \quad q_{E}=2\left(\frac{16370483137}{53542288800 \sqrt{2}}-\frac{\ln (2)}{2 \sqrt{2}}\right), \\
& p_{L_{z}}=\frac{4 \sqrt{2}}{5}, \quad q_{L_{z}}=2\left(\frac{1613849 \sqrt{2}}{1616615}-2 \sqrt{2} \ln (2)\right) .
\end{aligned}
$$

The fitting functions (29)-(31) may be similarly expanded near $r_{p}=r_{\mathrm{UCO}}=4 M$

$$
\frac{M}{m} \Delta X=-A_{X} \ln \left(\frac{r_{p}}{M}-4\right)+A_{X} \ln \left(2 b_{X}\right)+O\left(\frac{r_{p}}{M}-4\right) .
$$

In this, $X$ once again refers to either $E$ or $L_{z} / M$, and $N_{E}=$ 7, $N_{L_{z}}=4$. Equating (40) and (43), one obtains the coefficients of the fitting function given earlier (30) and (32).

A similar analysis can be performed for orbits of arbitrary eccentricity, and is described in Sec. 1 of the appendix. The exact expressions are somewhat cumbersome and we recommend using the fitting function in most applications, since this performs extremely well. The exact expressions have been included for completeness, and to help explain why the fitting function works.

\section{E. Hyperbolic captures}

In this paper we mostly focus on parabolic orbits, which serve as a useful model for all orbits which are likely to lead to sources of interest to LISA, since such orbits will always initially have eccentricities very close to 1 . However, objects can also be captured from orbits with $e>$ 1. In such cases the orbit is unbound, but may ultimately inspiral if it makes a close approach to the central black hole and loses enough energy and angular momentum in doing so to become bound. Our results suggest that if the angular momentum of this orbit is low (close to the minimum $L_{z}=4$ ), then the scattered body will become bound if it is on an orbit whose energy $E$ is such that $E^{2}-1<$ $m / M$ roughly speaking, where $m / M$, the mass ratio, is small. For larger angular momenta the amount of excess energy which can be radiated away on the first pass is smaller, and so the orbital energy must be even closer to unity for the body to become bound. Figure 8 shows which hyperbolic orbits can lead to captures, for low orbital angular momenta. The energy and angular momentum lost to gravitational waves by a particle on a hyperbolic orbit are given by the same Eqs. (A3) and (A4) that apply to bound orbits, just by inserting $e>1$ consistent with the definitions (12) and (13). Figure 8 was generated by using Eqs. (12) and (13) in conjunction with Eq. (A3) to write $\Delta E=(m / M) F_{E}\left(E, L_{z}\right)$ for hyperbolic orbits. Points on the curves obey the equation

$$
E-1=-\frac{m}{M} F_{E}\left(E, L_{z}\right)
$$

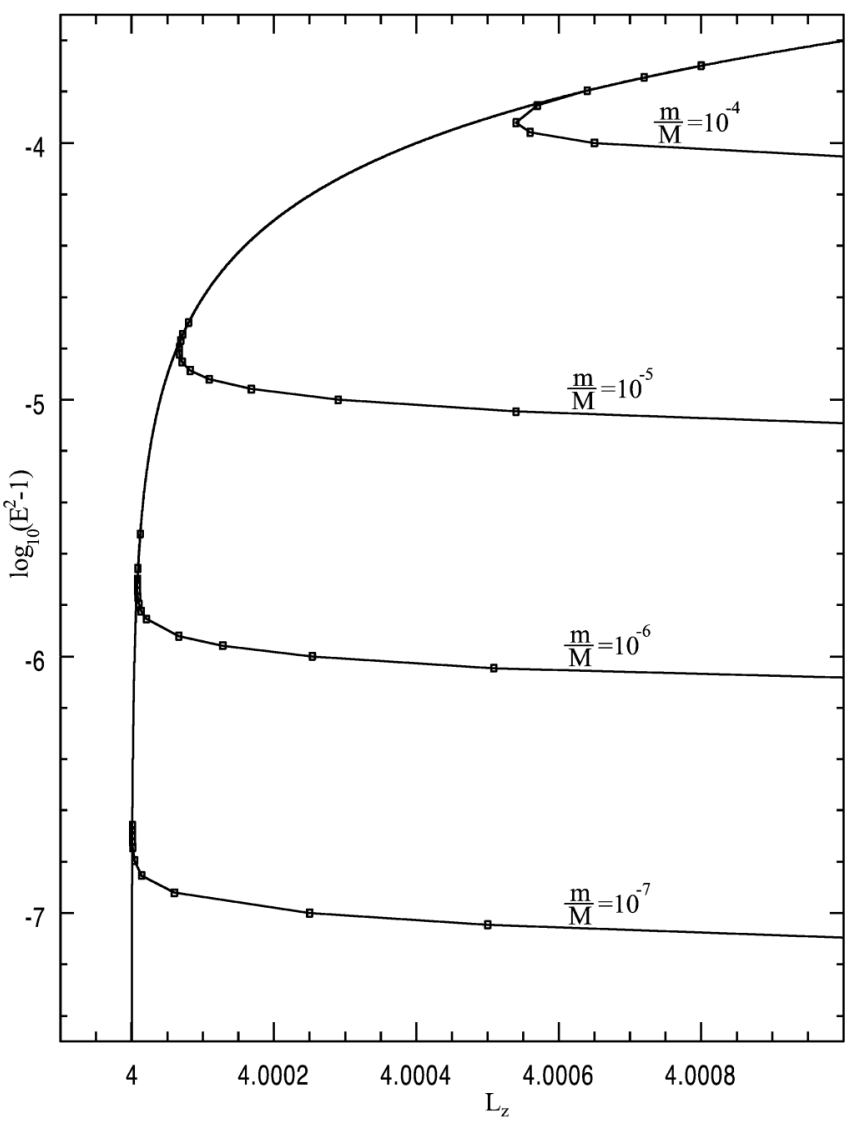

FIG. 8. "Hyperbolic" orbits (i.e., orbits with $E>1$ ) that are captured after one close encounter with the black hole, for various mass ratios. Orbits whose energy and angular momentum place them above and to the right of the line for a given mass ratio remain unbound and are not captured. The line which begins at bottom left and curves around to top right in the figure is the separatrix line separating unstable plunging orbits (to its left) from stable orbits.

Fixing the energy, the angular momentum solution to (44) is obtained by iteration.

This figure indicates that there is another type of orbit which becomes bound after the first pass - those that are close to the separatrix. If the orbit is very close to the plunge line, it will also lose enough energy to become bound even if it has much more energy than $E^{2}-1=$ $m / M$. The reason seems obvious after a glance at Fig. 1. If the energy of the scattered body is sufficiently close to 1 then it is close enough to the potential well in which bound orbits exist to lose sufficient energy on one pass to fall into the well. If the energy and angular momentum of the orbit are such that the particle's energy places it at the maximum of the potential, then the particle "whirls" around the central black hole at the radius of the potential maximum. The scattered body thus spends an abnormally long time near periastron and hence radiates an unusually large amount of energy, enough to become bound. Of course these bodies will generally plunge rather soon after capture because the amount of angular momentum radiated will 
also decrease the height of the potential barrier. In fact many of these orbits will plunge on their first pass, having dissipated enough angular momentum to shrink the potential barrier so they pass over it into the plunging region beyond. One has to keep in mind that our adiabatic approximation breaks down as one approaches the line separating stable from plunging orbits (depending on the mass ratio), so that we can give no definitive picture of what occurs in this regime except to say that the general behavior is probably correct. Readers interested in the transition from inspiral to plunge should consult [21].

Orbits that are scattered close to the separatrix line are "captured," in the sense that they either plunge or become bound. Particles which are close to being parabolic orbits are also captured and may serve to modestly increase the capture rate for LISA. Particles passing very near to the black hole must thus pass between Scylla and Charybdis [26]. If they pass too close to Scylla, through having energy only marginally greater than 1 , then they are plucked from their unbound orbit by gravitational radiation reaction and end up in a bound orbit. If they approach with too great an energy for their angular momentum then they are sucked down by Charybdis and plunge into the black hole itself.

\section{RESULTS AND DISCUSSION}

As with Earth-based gravitational wave detectors like the Laser Interferometer Ground Observatory, theoretical predictions of source event rates and signal characteristics for LISA will play an important role in the successful operation of the observatory. Up to now, Newtonian-order estimates (like Peters and Mathews) have been widely relied upon to estimate waveforms and fluxes from extreme-mass ratio inspirals, even though much of what is of interest to LISA, even in the early stages of inspiral, occurs inside the region of relatively strong curvature close to the central black hole. The principle reason for this is simply ease of use. Even when accurate methods, such as the Teukolsky formalism and self-force calculations, prove capable of dealing with arbitrary orbits they may still be slow and cumbersome for many applications. This paper attempts to make available a range of techniques which combine ease of use with fairly robust accuracy over almost the whole inspiral of an extreme-mass-ratio binary. These results are of particular use for highly eccentric orbits, where frequency domain Teukolsky calculations perform poorly [10] and time domain codes have not yet been fully developed [23].

The key elements to take away from this study of the semirelativistic approximation are

(i) Simple analytic expressions to estimate the fluxes $\Delta E$ and $\Delta L_{z}$, suitable for use in computational endeavours.

(ii) The optimal choice of parameters with which to describe orbits which stray near the central black hole are the geodesic parameters $\left\{r_{p}, e\right\}$ rather than $\left\{E, L_{z}\right\}$ : the waveforms for orbits which have similar $\left\{r_{p}, e\right\}$ more closely match than orbits which have similar $\left\{E, L_{z}\right\}$ values.

This second point cannot be stressed enough, as it applies to treatments which use the semirelativistic approximation or Newtonian results; all approaches appear to be most accurate when the orbits are defined by the periapse distance $r_{p}$ and eccentricity $e$ rather than by energy and angular momentum. The reason is that when working in flat space relating the orbit to the curvedspacetime orbit with the same $r_{p}$ and $e$ gives much better agreement with the curved-spacetime fluxes derived by exact methods (Teukolsky methods) than with the fluxes from the curved-spacetime orbit with the same $E$ and $L_{z}$ as the flat-space orbit. This is one substantial improvement in accuracy (see Fig. 3) which can be made for no computational cost whatsoever.

To gain further improvements, the fitting function (A11) described in Sec. IIID 1 can be used for only a small additional computational cost. Using the coefficients presented here, we have seen that it can accurately reproduce the energy and angular momentum fluxes computed using the semirelativistic approximation. However, it also has more general applicability. Once sufficient data have been obtained by numerical solution of the Teukolsky equation, it should be possible to derive a good fit to that data using the same fitting ansatz. This will provide a more practical expression for use in astrophysical calculations.

In Sec. III C we made use of the semirelativistic results to estimate the inconsistency in kludge gravitational waveforms that are being used to scope out LISA data analysis [14-16]. These waveforms are constructed in a similar way to the semirelativistic fluxes described here, but the inspiral trajectory of the particle is computed independently of the waveforms using post-Newtonian results. We saw that the energy content of the gravitational waves can be as much as a factor of 3 greater than the energy lost by the particle orbit. This is an important point to bear in mind when interpreting results computed using these approximations.

The semirelativistic formalism presented here should find uses in computational problems where speed is of concern (e.g., large numerical simulations) and the role played by the central black hole is important to the dynamics of individual particles in the problem. Such problems of interest might include new simulations of star cluster evolution in galactic nuclei to estimate the LISA EMRI event rate, or supermassive black hole inspiral simulations which seek to use interactions with stellar populations as a source of dynamical friction to bring the large black holes into proximity. In a companion paper [22], we use the insight gained here, in conjunction with numerical results from solution of the Teukolsky equation [23], to compute improved expressions for the inspiral time scale of capture orbits. The resulting expressions can be easily included in 
simulations of stellar clusters [27-29] to improve estimates of capture rates.

The semirelativistic approximation can also be applied to estimate energy and angular momentum fluxes from objects orbiting Kerr black holes. The procedure is more complicated due to the inclusion of spin and lack of spherical symmetry. In particular, it is not clear how to evolve the third integral of the motion, the Carter constant, for Kerr inspirals. However, by identifying BoyerLindquist coordinates with flat-space spherical polar coordinates and constructing the corresponding flat-space quadrupole moment tensor in the manner employed here, estimates for the energy and angular momentum fluxes from Kerr orbits may still be obtained. Preliminary results suggest that such semirelativistic estimates improve over standard post-Newtonian results [30] for spinning black holes as well. To construct inspirals, the angular momentum and energy fluxes can be combined with kludge approximations for the evolution of the Carter constant $[14,15]$. This extension to Kerr will be described in a future paper.

\section{ACKNOWLEDGMENTS}

We would like to thank E. Sterl Phinney for initially suggesting this problem, and the members of the TAPIR group at Caltech for helpful discussions during the completion of this work. We also thank Kostas Glampedakis and Stanislav Babak for several helpful suggestions. S. L. L. and J.R.G. thank the Aspen Centre for Physics for their hospitality while the manuscript was being finished. This work was supported in part by NASA Grants No. NAG5-12834 (J. R. G., D. J. K.) and No. NAG5-10707 (J. R. G.). S. L. L. acknowledges support at Penn State from the Center for Gravitational Wave Physics, funded by the NSF under cooperative Agreement No. PHY 01-14375, as well as support from Caltech under LISA Contract No. PO 1217163.

\section{APPENDIX: ANALYTIC RESULTS FOR ARBITRARY ECCENTRICITY}

\section{Exact expressions}

For orbits of arbitrary eccentricity it is also possible to derive exact expressions for the loss in energy and angular momentum, which reduce to (35) and (37) in the parabolic case. This is accomplished by writing the energy and angular momentum lost as a sum of integrals of the form

$$
I_{n}=\int_{r_{p}}^{r_{a}} \frac{M^{n+1} d r}{r^{n} \sqrt{\left(r_{a}-r\right)\left(r-r_{p}\right)\left(r-r_{-}\right) r}} .
$$

By considering the derivative of $\sqrt{\left(r_{a}-r\right)\left(r-r_{p}\right)\left(r-r_{-}\right) r} / r^{n}$ and using results in [25], we deduce

$$
\begin{aligned}
I_{n}= & \left(\frac{n-1}{2 n-1}\right) I_{n-1}-\frac{M\left((1+e) r_{p}-\left(3+e^{2}\right) M\right)}{r_{p}^{2}(1+e)^{2}} \frac{(2 n-3)}{(2 n-1)} I_{n-2}+\frac{M^{2}(1-e)\left((1+e) r_{p}-4 M\right)}{r_{p}^{3}(1+e)^{2}}\left(\frac{n-2}{2 n-1}\right) I_{n-3} \\
I_{0}= & \frac{2 M}{r_{p}} \sqrt{\frac{(1-e)\left((1+e) r_{p}-4 M\right)}{(1+e)\left((1+e) r_{p}-2(3-e) M\right)} \mathbf{K}\left(\sqrt{\frac{4 e M}{\left((1+e) r_{p}-2(3-e) M\right)}}\right)} \\
I_{1}= & \frac{M\left((1+e) r_{p}-4 M\right)}{(1+e) r_{p}^{3}} \sqrt{\frac{(1-e)\left((1+e) r_{p}-4 M\right)}{(1+e)\left((1+e) r_{p}-2(3-e) M\right)}\left[r_{p} \mathbf{K}\left(\sqrt{\frac{4 e M}{\left((1+e) r_{p}-2(3-e) M\right)}}\right)\right.} \\
& \left.-\frac{r_{p}\left((1+e) r_{p}-2(3-e) M\right)}{(1+e) r_{p}-4 M} \mathbf{E}\left(\sqrt{\frac{4 e}{\left((1+e) r_{p}-2(3-e) M\right)}}\right)\right] .
\end{aligned}
$$

The functions $\mathbf{K}(k)$ and $\mathbf{E}(k)$ are the complete elliptic integrals of the first and second kinds (36). Using the recurrence relation (A2) we can express the energy and angular momentum lost in terms of these elliptic integrals. We find the expression for the energy loss to be

$$
\begin{aligned}
\frac{M}{m} \Delta E= & -\frac{16 M^{11}}{1673196525 r_{p}^{6}(1+e)^{19 / 2}\left(\left(r_{p}-2 M\right)\left((1+e) r_{p}-2(1-e) M\right)\right)^{5 / 2}} \\
& \times\left[\sqrt{(1+e) \frac{r_{p}}{M}-2(3-e)} \mathbf{E}\left(\sqrt{\frac{4 e M}{\left((1+e) r_{p}-2(3-e) M\right)}}\right) f_{1}\left(\frac{r_{p}}{M}, e\right)\right. \\
& \left.+\frac{(1+e)}{\sqrt{(1+e) \frac{r_{p}}{M}-2(3-e)}} \mathbf{K}\left(\sqrt{\frac{4 e M}{\left((1+e) r_{p}-2(3-e) M\right)}}\right) f_{2}\left(\frac{r_{p}}{M}, e\right)\right]
\end{aligned}
$$

where 


$$
\begin{aligned}
f_{1}(y, e)= & 4608(1-e)(1+e)^{2}\left(3+e^{2}\right)^{2}\left(2428691599+313957879 e^{2}+1279504693 e^{4}+63843717 e^{6}\right) \\
& +192(1+e)^{2}\left(908960573673-155717471796 e^{2}-88736969547 e^{4}-293676299040 e^{6}\right. \\
& \left.-195313674237 e^{8}-26635698156 e^{10}-346799201 e^{12}\right) y-384(1+e)^{3}(336063804453 \\
& \left.-53956775638 e^{2}-33318942522 e^{4}-92857670352 e^{6}-41764459155 e^{8}-2765710514 e^{10}\right) y^{2} \\
& +16(1+e)^{4}\left(3418907055555-580720618635 e^{2}-168432860626 e^{4}-606890963686 e^{6}\right. \\
& \left.-176495184865 e^{8}-3768291999 e^{10}\right) y^{3}-32(1+e)^{5}\left(510454645597-92175635794 e^{2}\right. \\
& \left.+26432814256 e^{4}-28250211070 e^{6}-5713846269 e^{8}\right) y^{4}+4(1+e)^{6}(1107402703901 \\
& \left.-174239346926 e^{2}+100957560852 e^{4}+3707280110 e^{6}-899162673 e^{8}\right) y^{5} \\
& -8(1+e)^{7}\left(143625217397-16032820010 e^{2}+4238287541 e^{4}+275190560 e^{6}\right) y^{6} \\
& +(1+e)^{8}\left(220627324753-14884378223 e^{2}-1210713997 e^{4}+14138955 e^{6}\right) y^{7} \\
& -8(1+e)^{9}\left(2922108518-46504603 e^{2}-2407656 e^{4}\right) y^{8}+3(1+e)^{10}\left(241579935+6314675 e^{2}\right. \\
& \left.-149426 e^{4}\right) y^{9}+4(1+e)^{11}\left(8608805-48992 e^{2}\right) y^{10}+2(1+e)^{12}\left(1242083-16320 e^{2}\right) y^{11} \\
& +184320(1+e)^{13} y^{12}+5120(1+e)^{14} y^{13}
\end{aligned}
$$

and

$$
\begin{aligned}
f_{2}(y, e)= & 3072(3-e)(3+e)\left(3+e^{2}\right)\left(7286074797-3299041125 e^{2}+792940362 e^{4}-1366777698 e^{6}\right. \\
& \left.-369698151 e^{8}-5932745 e^{10}\right)-384(1+e)\left(2989180413711-583867932642 e^{2}-131661872359 e^{4}\right. \\
& \left.-419423580924 e^{6}-194293515951 e^{8}-3390301442 e^{10}+1353430119 e^{12}\right) y \\
& +64(1+e)^{2}\left(14825178681327-2675442646782 e^{2}-728511901515 e^{4}-1837874368340 e^{6}\right. \\
& \left.-591999524567 e^{8}-1856757710 e^{10}+841581651 e^{12}\right) y^{2}-32(1+e)^{3}(14292163934541 \\
& -2666166422089 e^{2}-522582885086 e^{4}-1347373382962 e^{6}-307066297439 e^{8} \\
& \left.-1675056789 e^{10}\right) y^{3}+16(1+e)^{4}\left(9557748374919-1917809903861 e^{2}-24258045506 e^{4}\right. \\
& \left.-511875047746 e^{6}-86779453317 e^{8}-462078345 e^{10}\right) y^{4}-8(1+e)^{5}(5390797838491 \\
& \left.-990602472036 e^{2}+161182699002 e^{4}-89978894004 e^{6}-11363685245 e^{8}\right) y^{5} \\
& +4(1+e)^{6}\left(2857676457065-351292910556 e^{2}+79840371470 e^{4}-2670080940 e^{6}\right. \\
& \left.-463345647 e^{8}\right) y^{6}-2(1+e)^{7}\left(1249768416047-79903103833 e^{2}+12179840133 e^{4}\right. \\
& \left.+482157413 e^{6}\right) y^{7}+(1+e)^{8}\left(363565648057-10040939153 e^{2}-318841465 e^{4}+14611473 e^{6}\right) y^{8} \\
& -2(1+e)^{9}\left(13862653487-100645509 e^{2}-11015842 e^{4}\right) y^{9}+(1+e)^{10}\left(518128485+16345427 e^{2}\right. \\
& \left.-421398 e^{4}\right) y^{10}+16(1+e)^{11}\left(1220639-13448 e^{2}\right) y^{11}+2(1+e)^{12}\left(689123-18880 e^{2}\right) y^{12} \\
& +153600(1+e)^{13} y^{13}+5120(1+e)^{14} y^{14} .
\end{aligned}
$$

The angular momentum lost is similarly given by

$$
\begin{aligned}
\frac{d L_{z}}{m}= & -\frac{16 M^{15 / 2}}{24249225(1+e)^{13 / 2} r_{p}^{7 / 2}\left(r_{p}-2 M\right)^{2}\left((1+e) r_{p}-2(1-e) M\right)^{2}} \\
& \times\left[\sqrt{(1+e) \frac{r_{p}}{M}-2(3-e)} \mathbf{E}\left(\sqrt{\frac{4 e M}{\left((1+e) r_{p}-2(3-e) M\right)}}\right) g_{1}\left(\frac{r_{p}}{M}, e\right)\right. \\
& \left.\left.+\frac{(1+e)}{\sqrt{(1+e) \frac{r_{p}}{M}-2(3-e)}}\right) g_{2}\left(\frac{r_{p}}{M}, e\right)\right]
\end{aligned}
$$

where 


$$
\begin{aligned}
g_{1}(y, e)= & 169728(1-e)(1+e)^{2}\left(279297+219897 e^{2}+106299 e^{4}+9611 e^{6}\right)-384(1+e)^{2}(192524061 \\
& \left.-13847615 e^{2}-36165965 e^{4}-20710173 e^{6}-588532 e^{8}\right) y+192(1+e)^{3}\left(235976417+13109547 e^{2}\right. \\
& \left.-3369705 e^{4}-3292707 e^{6}\right) y^{2}-16(1+e)^{4}\left(813592799+112906199 e^{2}+53843933 e^{4}+602061 e^{6}\right) y^{3} \\
& +16(1+e)^{5}\left(87491089+7247482 e^{2}+4608349 e^{4}\right) y^{4}+8(1+e)^{6}\left(9580616+6179243 e^{2}\right. \\
& \left.-92047 e^{4}\right) y^{5}-4(1+e)^{7}\left(3760123+272087 e^{2}\right) y^{6}-(1+e)^{8}\left(1168355-35347 e^{2}\right) y^{7} \\
& -71792(1+e)^{9} y^{8}-4120(1+e)^{10} y^{9}
\end{aligned}
$$

and

$$
\begin{aligned}
g_{2}(y, e)= & 339456(3-e)(3+e)\left(93099-10213 e^{2}-18155 e^{4}-10551 e^{6}-420 e^{8}\right)-1536(1+e)(319648410 \\
& \left.-35712133 e^{2}-33099777 e^{4}-11272311 e^{6}+457187 e^{8}\right) y+128(1+e)^{2}\left(2706209781-45415294 e^{2}\right. \\
& \left.-103634296 e^{4}-34056010 e^{6}-130293 e^{8}\right) y^{2}-32(1+e)^{3}\left(3895435659+212168215 e^{2}\right. \\
& \left.+4641265 e^{4}-15197651 e^{6}\right) y^{3}+16(1+e)^{4}\left(1396737473+123722895 e^{2}+27602127 e^{4}\right. \\
& \left.-465119 e^{6}\right) y^{4}-16(1+e)^{5}\left(78148621+3035912 e^{2}+3130827 e^{4}\right) y^{5}-16(1+e)^{6}(8005570 \\
& \left.+1485159 e^{2}-47943 e^{4}\right) y^{6}+2(1+e)^{7}\left(4015181+601959 e^{2}\right) y^{7}+(1+e)^{8}\left(737603-39467 e^{2}\right) y^{8} \\
& +47072(1+e)^{9} y^{9}+4120(1+e)^{10} y^{10} .
\end{aligned}
$$

The limit $r_{p} \rightarrow \infty$ corresponds to the argument of the elliptic integrals approaching zero. Using series expansions of the elliptic integrals about $k=0$ [24], we find

$$
\begin{aligned}
\frac{M}{m} \Delta E \approx & -\frac{64 \pi}{5} \frac{1}{(1+e)^{7 / 2}}\left(1+\frac{73}{24} e^{2}+\frac{37}{96} e^{4}\right)\left(\frac{r_{p}}{M}\right)^{-7 / 2}-\frac{64 \pi}{5} \frac{1}{(1+e)^{9 / 2}}\left(1+\frac{31}{8} e^{2}+\frac{65}{32} e^{4}+\frac{1}{6} e^{6}\right)\left(\frac{r_{p}}{M}\right)^{-9 / 2} \\
& +O\left(\left(\frac{r_{p}}{M}\right)^{-11 / 2}\right) \\
\frac{\Delta L_{z}}{m} \approx- & -\frac{64 \pi}{5} \frac{1}{(1+e)^{2}}\left(1+\frac{7}{8} e^{2}\right)\left(\frac{r_{p}}{M}\right)^{-2}-\frac{192 \pi}{5} \frac{1}{(1+e)^{3}}\left(1+\frac{35}{24} e^{2}+\frac{1}{4} e^{4}\right)\left(\frac{r_{p}}{M}\right)^{-3}+O\left(\left(\frac{r_{p}}{M}\right)^{-4}\right) .
\end{aligned}
$$

The leading order terms agree with the Keplerian results (22) and (23) [3], as expected. In the limit $r_{p} \rightarrow r_{\mathrm{UCO}}=$ $2(3+e) /(1+e)$, the argument of the elliptic integrals approaches 1 . The elliptic integrals diverge logarithmically in this limit, and we may expand them as in Eqs. (38) and (39).

On substitution of these expansions into (A3) and (A4), we find the asymptotic form of $\Delta E$ and $\Delta L_{z}$ to be

$$
\frac{M}{m} \Delta X \approx p_{X} \ln \left(\frac{r_{p}}{M}-\frac{2(3+e)}{1+e}\right)+q_{X}+O\left(\left(\frac{r_{p}}{M}-\frac{2(3+e)}{1+e}\right) \ln \left(\frac{r_{p}}{M}-\frac{2(3+e)}{1+e}\right)\right)
$$

As before, $X$ refers to either $E$ or $L_{z} / M$. The coefficients $p_{X}$ and $q_{X}$ are functions of eccentricity

$$
p_{E}=\frac{4(1+e)^{7 / 2}}{5 \sqrt{e}(3+e)^{3}}
$$




$$
\begin{aligned}
q_{E}= & 4 \sqrt{e}\left(126493657290+548139181590 e+1030019780790 e^{2}+1139255611065 e^{3}+838466930873 e^{4}\right. \\
& +401719467929 e^{5}+98700067049 e^{6}+6236043751 e^{7}+2856045401 e^{8}-177251547 e^{9} \\
& \left.-1203124043 e^{10}+316812556 e^{11}+109455696 e^{12}-88995328 e^{13}\right) /\left(1673196525(1+e)^{5 / 2}(3+e)^{6}\right) \\
& -\frac{4(1+e)^{7 / 2}(\ln (64)-\ln (1+e)+\ln (e))}{5 \sqrt{e}(3+e)^{3}}
\end{aligned}
$$

$$
\begin{aligned}
& p_{L_{z}}=\frac{8 \sqrt{2}(1+e)^{2}}{5(3+e)^{3 / 2} \sqrt{e}} \\
& q_{L_{z}}= \frac{16 \sqrt{2}(1+e)^{2}}{24249225(3+e)^{3 / 2} \sqrt{e}}\left(\frac { e } { ( 1 + e ) ^ { 4 } ( 3 + e ) ^ { 2 } } \left(174594420+523783260 e+557732175 e^{2}+241337525 e^{3}\right.\right. \\
&\left.+44249062 e^{4}+11244922 e^{5}-2993241 e^{6}-1809123 e^{7}+1328784 e^{8}-172744 e^{9}\right) \\
&\left.-\frac{4849845(6 \ln (2)-\ln (1+e)+\ln (e))}{2}\right) .
\end{aligned}
$$

\section{Fitting functions}

We can use the exact expressions (A3) and (A4) to derive fitting functions to approximate our results. Following the same argument used in the parabolic case, a functional form like (29) should capture the main features of the problem, but the coefficients are now functions of eccentricity, and we replace the parabolic value of the UCO-4M-with the value appropriate to other eccentricities. The general ansatz is

$$
\begin{aligned}
\frac{M}{m} \Delta E= & \left(\sum_{n=0}^{N} A_{n}^{E}(e)\left(\frac{M\left((1+e) r_{p}-2(3+e) M\right)}{(1+e) r_{p}^{2}}\right)^{n}\right) \cosh ^{-1}\left[1+B_{0}^{E}\left(\frac{2(3+e) M}{(1+e) r_{p}}\right)^{N_{E}-1} \frac{(1+e) M}{(1+e) r_{p}-2(3+e) M}\right] \\
& +\frac{M^{N_{E} / 2}\left((1+e) r_{p}-2(3+e) M\right)}{(1+e) r_{p}^{1+\left(N_{E} / 2\right)}} \sum_{n=0}^{N} C_{n}^{E}\left(\frac{M\left((1+e) r_{p}-2(3+e) M\right)}{(1+e) r_{p}^{2}}\right)^{n} \\
& +\frac{M^{1+\left(N_{E} / 2\right)}\left((1+e) r_{p}-2(3+e) M\right)}{(1+e) r_{p}^{2+\left(N_{E} / 2\right)}} \sum_{n=0}^{N-1} B_{n+1}^{E}\left(\frac{M\left((1+e) r_{p}-2(3+e) M\right)}{(1+e) r_{p}^{2}}\right)^{n} .
\end{aligned}
$$

Successive terms in the fit are given by matching consecutive orders in an expansion about $r_{p}=2(3+e) /(1+e)$ and as $r_{p} \rightarrow \infty$ in the way described in Sec. IIID 1 for the parabolic case. To illustrate, the lowest order $(N=0)$ expansion coefficients may be determined from the $\Delta E$ and $\Delta L_{z}$ expansions (A5)-(A10) as follows:

$$
\begin{gathered}
A_{0}^{X}(e)=-p_{X}(e), \quad B_{0}^{X}(e)=\frac{1}{2} \exp \left(\frac{q_{X}(e)}{A_{X}(e)}\right), \\
C_{0}^{E}(e)=-\frac{64 \pi}{5} \frac{1}{(1+e)^{7 / 2}}\left(1+\frac{73}{24} e^{2}+\frac{37}{96} e^{4}\right)-A_{0}^{E}(e) \sqrt{2 B_{0}^{E}(e)}\left(\frac{2(3+e)}{(1+e)}\right)^{3}, \\
C_{0}^{L_{z}}(e)=-\frac{64 \pi}{5} \frac{1}{(1+e)^{2}}\left(1+\frac{7}{8} e^{2}\right)-A_{0}^{L_{z}}(e) \sqrt{2 B_{0}^{L_{z}}(e)}\left(\frac{2(3+e)}{(1+e)}\right)^{3 / 2} .
\end{gathered}
$$

Our main focus is on orbits that are nearly parabolic, with $e \approx 1$. We therefore expand these expressions about $e=1$ to 
obtain

$$
\begin{aligned}
& A_{0}^{E}(e)=-\frac{1}{5 \sqrt{2}}+\frac{1}{10 \sqrt{2}}(1-e)-\frac{1}{160 \sqrt{2}}(1-e)^{2}+O\left((1-e)^{3}\right) \\
& B_{0}^{E}(e)=0.752091-0.0949439(1-e)+0.0918458(1-e)^{2}+O\left((1-e)^{3}\right) \\
& C_{0}^{E}(e)=-4.63464+1.63944(1-e)+0.327505(1-e)^{2}+O\left((1-e)^{3}\right) \\
& A_{0}^{L_{z}}(e)=-\frac{4 \sqrt{2}}{5}+\frac{1}{5 \sqrt{2}}(1-e)-\frac{7}{80 \sqrt{2}}(1-e)^{2}+O\left((1-e)^{3}\right) \\
& B_{0}^{L_{z}}(e)=1.31899-0.126207(1-e)+0.392812(1-e)^{2}+O\left((1-e)^{3}\right) \\
& C_{0}^{L_{z}}(e)=-4.1491+1.71517(1-e)-0.128645(1-e)^{2}+O\left((1-e)^{3}\right) .
\end{aligned}
$$

The expansion of the $B_{0}^{X}$,s and $C_{0}^{X}$,s may also be written down precisely. However, the expressions are extremely complicated, which is why the numerical values of these coefficients have been quoted instead. Higher order fitting functions may be obtained by matching more terms in the expansions of $\Delta E$ and $\Delta L_{z}$, as described earlier. For completeness, we quote here the remaining coefficients of the $N=2 \mathrm{fit}$, once again expanded to quadratic order about the parabolic case

$$
\begin{aligned}
& A_{1}^{E}(e)=-0.282843(1-e)+0.0353553(1-e)^{2}+O\left((1-e)^{3}\right) \\
& B_{1}^{E}(e)=-103.215+39.6287(1-e)+38.3325(1-e)^{2}+O\left((1-e)^{3}\right) \\
& C_{1}^{E}(e)=69.1683-0.682028(1-e)-28.7945(1-e)^{2}+O\left((1-e)^{3}\right) \\
& A_{2}^{E}(e)=-1.20797-2.31872(1-e)-2.15134(1-e)^{2}+O\left((1-e)^{3}\right) \\
& B_{2}^{E}(e)=727.515+1570.89(1-e)+1139.13(1-e)^{2}+O\left((1-e)^{3}\right) \\
& C_{2}^{E}(e)=-439.378-1223.38(1-e)-862.812(1-e)^{2}+O\left((1-e)^{3}\right) \\
& A_{1}^{L_{z}}(e)=-0.565685(1-e)+0.494975(1-e)^{2}+O\left((1-e)^{3}\right) \\
& B_{1}^{L_{z}}(e)=-53.4491+4.38709(1-e)+0.469838(1-e)^{2}+O\left((1-e)^{3}\right) \\
& C_{1}^{L_{z}}(e)=25.4129+16.7694(1-e)-7.06419(1-e)^{2}+O\left((1-e)^{3}\right) \\
& A_{2}^{L_{z}}(e)=3.9598(1-e)-4.80833(1-e)^{2}+O\left((1-e)^{3}\right) \\
& B_{2}^{L_{z}}(e)=29.7857+167.281(1-e)+66.0607(1-e)^{2}+O\left((1-e)^{3}\right) \\
& C_{2}^{L_{z}}(e)=15.1726-131.512(1-e)-26.8611(1-e)^{2}+O\left((1-e)^{3}\right) .
\end{aligned}
$$

[1] J. R. Gair, L. Barack, T. Creighton, C. Cutler, S. L. Larson, E.S. Phinney, and M. Vallisneri, Classical Quantum Gravity 21, S1595 (2004).

[2] F. D. Ryan, Phys. Rev. D 56, 1845 (1997); 56, 7732 (1997).

[3] P. C. Peters and J. Mathews, Phys. Rev. 131, 435 (1963).

[4] P. C. Peters, Phys. Rev. 136, B1224 (1964).

[5] S. Teukolsky, Astrophys. J. 185, 635 (1973).

[6] W. Press and S. Teukolsky, Astrophys. J. 185, 649 (1973).

[7] W. Press and S. Teukolsky, Astrophys. J. 193, 443 (1974).

[8] C. Cutler, D. Kennefick, and E. Poisson, Phys. Rev. D 50, 3816 (1994).

[9] S. A. Hughes, Phys. Rev. D 61, 084004 (2000).
[10] K. Glampedakis and D. Kennefick, Phys. Rev. D 66, 044002 (2002).

[11] S. Drasco and S. Hughes, Phys. Rev. D 69, 044015 (2004).

[12] S. A. Hughes, S. Drasco, E. E. Flanagan, and J. Franklin, Phys. Rev. Lett. 94, 221101 (2005).

[13] R. Ruffini and M. Sasaki, Prog. Theor. Phys. 66, 1627 (1981).

[14] K. Glampedakis, S. A. Hughes, and D. Kennefick, Phys. Rev. D 66, 064005 (2002).

[15] J.R. Gair and K. Glampedakis (unpublished).

[16] T. Creighton, J. R. Gair, S. A. Hughes, and M. Vallisneri (unpublished).

[17] C. W. Misner, K. S. Thorne, and J. A. Wheeler, Gravitation (Freeman, New York, 1973). 
[18] E. Poisson, Living Rev. Relativity 7, 6 (2004), http:// www.livingreviews.org/lrr-2004-6.

[19] L. Barack and C. Cutler, Phys. Rev. D 70, 122002 (2004).

[20] T. Tanaka, M. Shibata, M. Sasaki, H. Tagoshi, and T. Nakamura, Prog. Theor. Phys. 90, 65 (1993); 71, 79 (1984).

[21] R. O'Shaughnessy, Phys. Rev. D 67, 044004 (2003).

[22] J.R. Gair, D. Kennefick, and S.L. Larson, astro-ph/ 0508275 .

[23] K. Martel, Phys. Rev. D 69, 044025 (2004).

[24] M. Abramowitz and I. Stegun, Handbook of Mathematical Functions (Dover, New York, 1964).

[25] I.S. Gradshteyn and I. M. Ryshik, Table of Integrals, Series and Products (Academic, London, 1994), 5th ed.
[26] In The Odyssey Scylla and Charybdis were monsters who guarded opposite sides of a narrow strait through which ships must pass on their way from Troy in Asia Minor to Ithaca in Greece. Scylla was a six-headed reptile who plucked sailors from the decks of their ships as they passed, while Charybdis was an undersea monster which created a great whirlpool by its sucking in of seawater, the whirlpool causing ships to be pulled under if they strayed too near.

[27] M. Freitag, Classical Quantum Gravity 18, 4033 (2001).

[28] M. Freitag and W. Benz, Astron. Astrophys. 394, 345 (2002).

[29] M. Freitag, Astrophys. J. 583, L21 (2003).

[30] F. D. Ryan, Phys. Rev. D 53, 3064 (1996). 